\title{
PHYSIOLOGIE DU DÉPLACEMENT \\ DES SPERMATOZOIDES \\ DANS LES VOIES GÉNITALES FEMELLES \\ CHEZ LA BREBIS ET LA VACHE (suite)
}

PAR

\section{DAUZIER}

Station de recherches de Physiologie animale Centre national de Recherches zootechniques

Jouy-en-Josas

\section{Chapitre IV}

\section{MÉCANISMES PHYSIOLOGIQUES \\ DE LA MONTÉE DES SPERMATOZOIDES DANS LE TRACTUS GÉNITAL FEMELLE \\ (Etude chez la Brebis).}

La progression des spermatozoïdes du vagin vers les trompes de Fallope, peut être due, soit à leur motilité, le tractus femelle n'intervenant que pour leur fournir un milieu favorable, soit à des facteurs propres à la femelle : contraction des parois utérines ou tubaires, mouvements ciliaires, force de succion due au mouvement des viscères, etc., soit à la combinaison des possibilités des spermatozoïdes à celles offertes par le tractus.

Les auteurs ayant rapporté une montée lente des spermatozoïdes, de l'ordre de plusieurs heures, ont été enclins à voir dans la seule motilité des gamètes mâles, l'élément déterminant de leur progression.

Au contraire ceux qui ont cru observer un transit rapide ont recherché dans les facteurs extérieurs aux spermatozoïdes, les causes essentielles de cette progression rapide ; pour certains la motilité des gamètes n'interviendrait même pas.

Ayant défini la vitesse de montée des spermatozoïdes et montré la fantaisie de certaines affirmations, nous avons analysé le rôle respectif de chaque facteur probable à chacun des différents niveaux du tractus : cervix, cornes utérines, jonction utéro-tubaire, trompes de Fallope. Compte tenu des différences anatomiques du tractus, on est en droit de penser que les mécanismes assurant la progression des spermatozoïdes peuvent différer d'un point à l'autre du tractus. 


\section{I. - ÉTUdE DE LA MONTÉE DES SPERMATOZOIDES DE BÉLIER DANS LE CERVIX DE LA BREBIS}

\section{Documentation bibliographique.}

Dès I 872 , I 1 oT'T indique les différents facteurs susceptibles de déter-. miner les transports des spermatozoïdes dans le cervix :

- force de l'éjaculat,

- action du pénis comparable à celle d'un piston,

- contractions des parois du tractus génital,

- action de succion de l'utérus,

- attraction capillaire dans la lumière du cervix,

- action ciliaire de l'épithélium cervical,

- mouvement des spermatozoïdes eux-mêmes.

PARKER (I93I) y ajoute l'action de la pression mutuelle des viscères.

Pour HEAPE (I898), la plus grande part dans la progression des spermatozoïdes au travers du col utérin de la Lapine, revient à l'action de succion de l'utérus, consécutive à l'excitation des tissus érectiles de la vulve.

WaLTON (1930) croit que la seule motilité des spermatozoïdes explique leur montée, opinion déjà formulée par HokHne (IgII) et MarsHALL (I922). Il en voit la preuve dans le fait qu'en injectant du liquide coloré dans le vagin d'une Lapine avant le coït, il n'en trouve aucune trace ultérieurement dans l'utérus. PARKER (I93r) critique cette expérience, en alléguant que le dépôt de liquide dans le vagin, avant l'accouplement, empêche une réponse normale des parois utérines de la Lapine. Pour PARKER (I93I), c'est en effet à une action active du tractus génital femelle qu'est dû le transport des spermatozoïdes qu'il aurait dénombrés dans l'utérus de Lapines I minute 50 secondes après le coït. Se basant sur le fait que les spermatozoïdes de Lapin progressent $i n$ vitro à une vitesse de $0,05 \mathrm{~mm} / \mathrm{sec}$ (LLOYD-JONES, HAYs, Igr 8 ; PARKER, I93I), il en déduit que les spermatozoïdes progressant par eux-mêmes, auraient dû mettre, au moins 4 minutes, en se déplaçant en ligne droite, sinon 8 minutes pour atteindre l'utérus. En fait, il est illusoire de vouloir comparer la progression des gamètes mâles in vivo et in vitro, les conditions étant totalement différentes.

Talmey (I9I7) a décrit un spasme cervical chez la Femme mais pour DICKInson (I93I) les mouvements du cervix ne sont pas essentiels à la progression des spermatozoïdes.

BLANDAU (I945) cherchant à expliquer le mécanisme de progression des spermatozoïdes de Rat, émis dans le vagin, constate que le cervix est dans un état de contraction maximum, chez la Rate, en moyenne pendant $\mathrm{r} 2,8 \mathrm{~h}$, soit, de $4,3 \mathrm{~h}$ avant à $8,5 \mathrm{~h}$ après le début de l'œstrus. 
Il essaie de mettre, expérimentalement, en évidence un relâchement au moment du coït, mais n'observe pas de mouvement de liquide, entre les cornes utérines, qui en contiennent d'abondantes quantités (LoNG et Evans, I922), (LeONard, I950), et le vagin. Par contre, il détermine le rôle important joué par le " plug " ou bouchon vaginal ; les spermatozoïdes de Rat, dont les glandes séminales et coagulantes sont ligaturées, n'entrent pas dans l'utérus, alors que dès la formation du bouchon, ils y progressent en grand nombre. STEINACH (I894) et WaLKer (I9II) ont montré, d'ailleurs, que l'ablation de ces glandes diminue beaucoup la fertilité ; la suppression concomitante de la prostate entrainant la stérilité.

On ne peut donc avoir d'opinion sur le mécanisme qui permet aux spermatozoïdes de pénétrer en grand nombre dans le cervix après le coit.

\section{$2^{\circ}$ Résultats personnels.}

La projection des spermatozoïdes à l'intérieur même du col utérin, lors du coï, pouvant intervenir comme mécanisme de progression des gamètes dans le cervix, 3 brebis ont été sacrifiées immédiatement après l'accouplement avec un bélier fécond. Des spermatozoïdes très mobiles n'ont été trouvés (tableau XIII) qu'à l'entrée du cervix, aucun d'entre eux n'avant pénétré dans le canal cervical.

Tableau XIII

\begin{tabular}{|c|c|c|c|c|c|c|c|}
\hline \multirow[b]{2}{*}{$\begin{array}{l}\text { Numéro } \\
\text { des Brebis }\end{array}$} & \multirow{2}{*}{$\begin{array}{l}\text { Intervalle } \\
\text { insémination- } \\
\text { abattage }\end{array}$} & \multicolumn{6}{|c|}{ Nombre de spermatozoïdes } \\
\hline & & Vagin & $\begin{array}{l}\text { Gutrée } \\
\text { Cervis }\end{array}$ & $\begin{array}{c}\text { r cm } \\
\text { intérieur } \\
\text { Cervix }\end{array}$ & $\begin{array}{l}\text { Milieu } \\
\text { Cervix }\end{array}$ & $\begin{array}{l}\text { Sortie } \\
\text { Cervix }\end{array}$ & Utérus \\
\hline $5^{25}$ & 30 secondes & $\infty$ & $\infty$ & $\circ$ & $\circ$ & $\circ$ & 0 \\
\hline 535 & I minute & $\infty$ & $\infty$ & 0 & 0 & 0 & 0 \\
\hline 554 & 30 secondes & $\infty$ & $\infty$ & c & o & o & $\circ$ \\
\hline
\end{tabular}

La présence des spermatozoïdes dans le col utérin ne pouvant être imputée à leur projection lors du rapprochement sexuel, nous avons vérifié, en utilisant nos techniques d'examen, si les spermatozoïdes de bélier morts, placés dans le vagin de la brebis, peurent progresser dans les voies femelles.

Les particules inertes (encre de Chine, carmin, carbone) ont été délaissées car il est difficile de discerner si l'on est en présence de particules retrouvées ou apportées, leur détermination étant beaucoup plus délicate que celle des spermatozoïdes colorés, comme nous l'ont montré quelques essais infructueux. Les particules inertes n'ont en outre pas nécessairement les mêmes dimensions et formes que les gamètes, ce qui peut éventuellement poser des problèmes particuliers dans l'étude de leur progression. 
Dans une première expérience, nous avons utilisé trois brebis inséminées au moyen d'un cathéter et d'un spéculum lumineux, avec $0,3 \mathrm{cc}$ de sperme préalablement tué par la chaleur. Les recherches des spermatozoïdes par la technique des frottis, chez ces brebis abattues 8 heures après l'insémination (le temps de 8 heures correspondant à la durée de montée des spermatozoïdes vivants) ont montré que les spermatozoïdes présents initialement dans la partie distale du vagin et à l'entrée du cervix, n'avaient pas progressé (tableau XIV).

\section{TABLEAU XIV}

\begin{tabular}{|c|c|c|c|c|c|c|c|}
\hline \multirow{2}{*}{ Traitement } & \multirow{2}{*}{$\begin{array}{l}\text { Numéro } \\
\text { Brebis }\end{array}$} & \multirow{2}{*}{$\begin{array}{l}\text { Intervalle } \\
\text { insémination- } \\
\text { abattage }\end{array}$} & \multicolumn{5}{|c|}{ Nombre de spermatozoides } \\
\hline & & & Vagin & $\begin{array}{l}\text { Entrée } \\
\text { Cervix }\end{array}$ & $\begin{array}{l}\text { Milieu } \\
\text { Cervix }\end{array}$ & $\begin{array}{l}\text { Sortie } \\
\text { Cervix }\end{array}$ & Utérus \\
\hline $\begin{array}{l}\text { Insémination : } \\
\text { Sperme tué par chaleur } 0,3 \mathrm{cc} \\
-\end{array}$ & $\begin{array}{l}450 \\
487 \\
435\end{array}$ & $\begin{array}{l}8 \text { heures } \\
8 \text { heures } \\
8 \text { heures }\end{array}$ & $\begin{array}{l}\infty \\
\infty \\
\infty\end{array}$ & $\begin{array}{l}\infty \\
99 \\
\infty\end{array}$ & $\begin{array}{l}\circ \\
\circ \\
\circ\end{array}$ & $\begin{array}{l}\circ \\
\circ \\
\circ\end{array}$ & $\begin{array}{l}\circ \\
\circ \\
\circ\end{array}$ \\
\hline $\begin{array}{l}\text { Insémination : } \\
\text { Sperme tué par chaleur o,3 cc } \\
\text { Iiquide spermatique o,3 } \mathrm{cc} \\
\text { Coit, Bélier vasectomisé }\end{array}$ & +46 & 8 heures & $\infty$ & 236 & $\circ$ & $\circ$ & $\circ$ \\
\hline - & $\begin{array}{l}+4 \mathrm{I} \\
483\end{array}$ & $\begin{array}{l}8 \text { heures } \\
8 \text { heures }\end{array}$ & $\begin{array}{l}\infty \\
\infty\end{array}$ & $\begin{array}{c}\infty \\
485\end{array}$ & $\begin{array}{l}\circ \\
\circ\end{array}$ & $\begin{array}{l}\circ \\
\circ\end{array}$ & : \\
\hline
\end{tabular}

Une excitation du cervix consécutive au coït ou à la présence de certaines substances dans le sperme vivant, fraîchement éjaculé, ou dans les sécrétions accessoires, pouvant jouer un rôle dans la montée des gamètes mâles, un deuxième groupe de trois brebis a été inséminé, avec 0,3 cc de sperme mort, puis $0,2 \mathrm{cc}$ de liquide spermatique, obtenu par centrifugation et filtrage de sperme frais sur filtres très fins, et enfin sailli par un bélier vasectomisé. Aucune montée de spermatozoïdes ne s'est produite chez ces Brebis sacrifiées 8 heures après l'insémination (tableau XIV).

Mais l'absence de spermatozoïdes après 8 heures pouvait être due à leur lyse ou à leur phagocytose rapides, d'autant plus qu'il s'agissait de gamètes morts. Aussi, quatre brebis, soumises au même traitement, ont-elles été abattues après un délai de $I$ heure. Les résultats sont identiques, aucun spermatozoide n'ayant été trouvé au-delà de la partie vaginale du col utérin.

Il semble donc évident que la motilité propre des spermatozoïdes soit un élément indispensable à leur progression, du vagin dans l'utérus.

L'utilisation d'un Bélier donneur d'un sperme de mauvaise qualité, présentant seulement des spermatozoïdes peu mobiles et de 80 à 95 p. Ioo de spermatozoïdes morts, accouplé avec 3 brebis en rut a permis de 
confirmer le rôle de la motilité des spermatozoïdes dans le franchissement du cervix. Une montée très faible des spermatozoïdes s'est produite, certains, après une heure, ayant atteint le milieu du cervix chez une brebis sur trois. ASDELL et SALISBURY (I94I) avaient obtenu des résultats semblables chez la Lapine, avec des spermatozoïdes devenus déficients et peu mobiles, après maintien des testicules de lapins, dans la paroi abdominale, pendant un certain temps.

Bien que le franchissement du cervix de Brebis se fasse grâce à la motilité des spermatozoïdes de Bélier, on peut penser que l'état du tractus femelle joue un rôle. Certains auteurs ont, d'ailleurs, étudié le passage du cervix par les spermatozoïdes en relation avec la viscosité du mucus cervical et sa perméabilité aux gamètes mâles. C'est ainsi que SEGuY et Vimeux (I934), Lamar, Shettrles, Delfs (I940), Ferin (1948), PomMERENKE et VIERGIVER (I946), ont montré que chez la Femme l'impottance des déplacements effectués par les spermatozoïdes varie en fonction inverse du degré de viscosité de la glaire cervicale, la perméabilité maximum aux spermatozoïdes se situant vers le milieu du cycle menstruel, c'est-à-dire vers le moment de l'ovulation (SEgur et Vimeux, I934); FERIN (I948) précise même que, dans la majorité des cas, en l'absence de "glaire filante", notamment dans la phase d'imprégnation progestinique, les spermatozoïdes ne semblent pas atteindre le fond de l'utérus. ABARBANEI (I946) signale que c'est juste avant le moment de l'ovulation que la viscosité du mucus est la plus faible, et sa pénétrabilité aux spermatozoïdes le plus élevée, les œestrogènes étant le facteur essentiel de production de cette glaire claire et filante.

BERRANG (I952) fait les mêmes observations chez la Vache : la distance que peuvent parcourir les spermatozoïdes est en relation inverse avec la viscosité du mucus. Par contre, certains auteurs contestent l'influence de l'activité ovarienne sur la progression de spermatozoïdes le long du tractus génital. STARKE (I949), puis GREEN et WINTERS (I935), SCHOTT et Phillips (I94I), Van Demark et Moellekr (I95I) ont conclu à des vitesses de montée des spermatozoïdes identiques aux différents moments du cycle sexuel. Ceci paraît étrange quand on connaît l'involution des voies femelles pendant le diœstrus et l'anœstrus de la Brebis, et les variations cycliques de la morphologie et de l'activité sécrétoire des différentes parties du tractus génital (McKkinZIE et TERrIL, I937).

C'est pourquoi nous avons repris cette étude sur 22 brebis de race Ile-de-France, accouplées en début, milieu et fin de diœstrus, et en anœstrus. Toutes les brebis furent abattues 8 heures ou I6 heures après l'accouplement, puisque comme nous l'avons vu chez la Brebis, les spermatozoïdes parviennent à la partie supérieure des trompes 8 heures après le coït chez les Brebis en œstrus. Une montée notable des spermatozoïdes dans le tractus génital de la Brebis ne se produit qu'au début du diœstrus. Dans 
ce cas nous n'avons trouvé que deux fois sur 8 des spermatozoïdes dans l'isthme, 8 heures et I6 heures après le coït : chez d'autres brebis ils n'avaient atteint que la base des cornes en 8 heures et le milieu en I6 heures (tableau XV).

Au milieu du dicestrus, vers le $8^{\mathrm{e}}$ jour du cycle, les spermatozoïdes ne dépassent pas le milieu du col, même I6 heures après l'accouplement. E,n fin de diøstrus, le niveau atteint est plus élevé au milieu des cornes : I brebis sur 6 - et le plus souvent, les spermatozoïdes sont présents dans lecorps de l'utérus et le milieu du cervix.

Enfin, chez les brebis accouplées pendant l'anœstrus, les spermatozoïdes ne sont jamais parvenus au-delà du corps de l'utérus ; le cervix n'a même été franchi que 2 fois sur 5 (tableau XV).

On doit rapprocher ces observations des résultats négatifs des recherches entreprises pour obtenir la fécondation de Brebis après injection d'hormone gonadotrope, soit en anœstrus, soit à n'importe quel moment du cycle sexuel, œstrus excepté (Murphez, Warwick, Casida et Mac Shan, I947; Boyarsky, Bayliss, Casida Meyer, I947; Thibault, ORTAVANT et LAPLAUD, I948). Le cervix constituerait un obstacle à la montée des spermatozoïdes, donc à la fécondation des œufs dont la ponte est aisément obtenue. MURPHeE, Black, OtTo, CASIDA (I95 I) confirment expérimentalement ce point de vue chez la Lapine, puisqu'ils montrent que le cervix constitue le principal obstacle à la fécondation des œufs pondus, lorsque le corps jaune est actif, pendant la pseudogestation de 1a Lapine: 1'insémination par voie vaginale donne 3,12 \% de féconda tion, tandis qu'en opérant par l'utérus, ces auteurs obtiennent $6 \mathrm{I} \%$ d'œufs fécondés.

La recherche des spermatozoïdes vivants aux différents niveaux des voies femelles en dehors de l'œestrus montre d'ailleurs (tableau XV) que la survie des spermatozoïdes est également affectée : elle est très courte, même dans le col, et elle n'est supérieure à 16 heures, chez la Brebis, qu'en début de diœstrus, alors que nous avons montré que les spermatozoïdes peuvent $y$ demeurer vivants plus de 3 jours pendant l'œestrus.

Leur résorption est également très rapide (tableau $\mathrm{XV}$ ) surtout en anœstrus, et en milieu de dicestrus : peu ou pas de spermatozoïdes furent dénombrés dans le tractus génital de 8 à I6 heures après le coït.

Ces faits soulignent le rôle actif du milieu cervical dans les possibilités de transport et de conservation des spermatozoïdes. Pendant l'ancestrus ou la phase lutéinique il constitue une barrière pratiquement infranchissable aux spermatozoïdes; la fécondation ne serait alors possible qu'en déposant la semence au-delà du col utérin. 


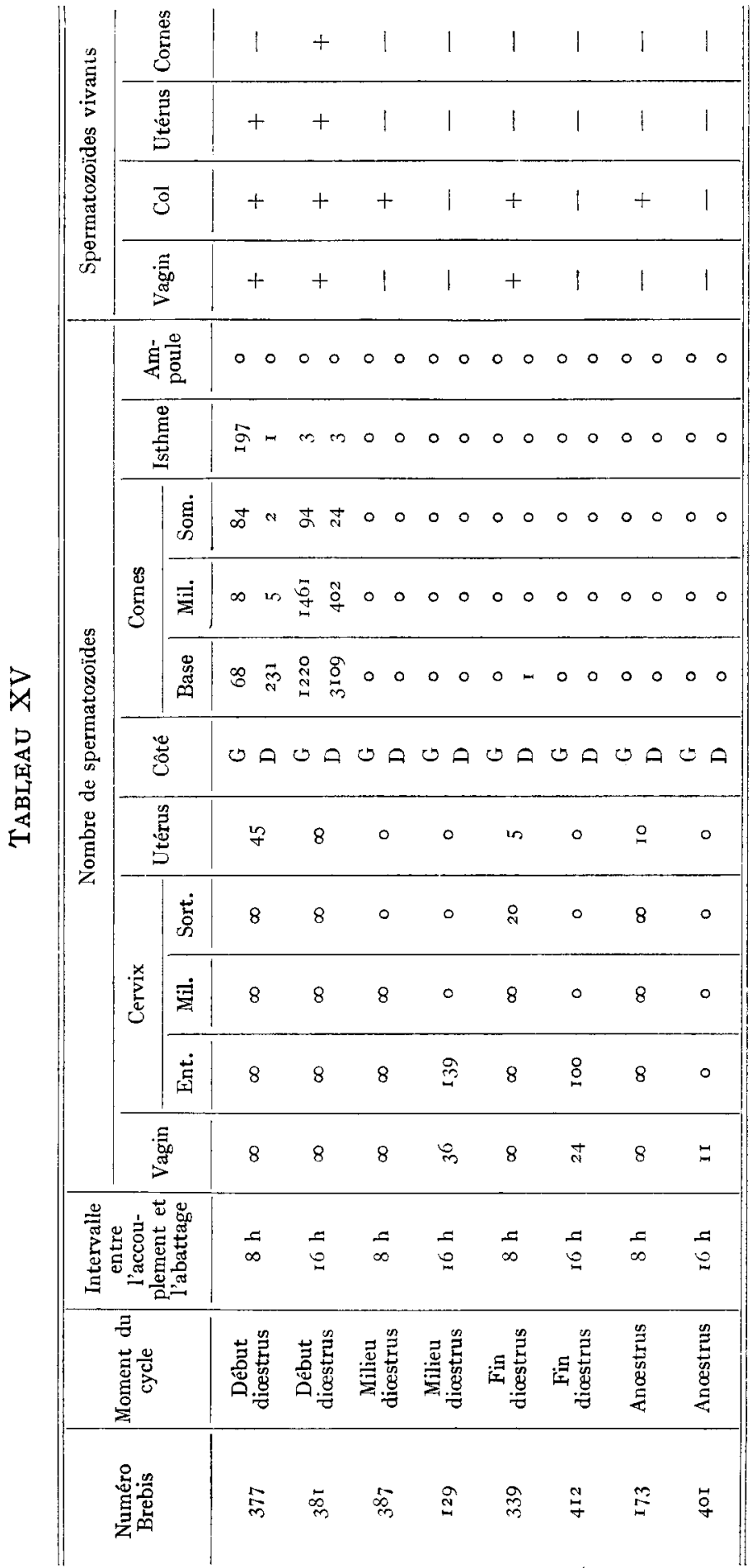




\section{II. - FACTEURS QUI CONDITIONNENT LA PROGRESSION DES SPERMATOZOIDES DE BELIER DANS L'UTÉRUS DE LA BREBIS}

\section{Bibliographie.}

L'étude des facteurs déterminant une progression des spermatozoïdes fait l'objet des mêmes contradictions bibliographiques pour les cornes utérines que pour le cervix, la primauté étant d'après les uns, à accorder à la motilité des gamètes mâles, d'après les autres, à un rôle actif du tractus génital femelle.

Marshal (I922) émet l'opinion que les spermatozoïdes de Lapin montent en progressant par leurs propres moyens, contre un courant dû aux mouvements ciliaires de l'épithélium utérin. Or, l'épithélium n'étant pas cilié (I,rm et Chaо (I927)), ces auteurs estiment que seul le rôle de leurs mouvements propres peut être retenu. PARKER (I93I) prétend aussi que les spermatozoïdes progressent dans l'utérus grâce à leur pouvoir locomoteur; il en voit la preuve dans les faits suivants : s'il injecte dans la partie distale de l'utérus des spermatozoïdes vivants il les retrouve dans la partie proximale 2 heures après, et réciproquement des spermatozoïdes déposés au bas des cornes sont présents au sommet après le même laps de temps; se référant à une vitesse de progression des spermatozoïdes in vitro, de $0,05 \mathrm{~mm} / \mathrm{sec}$, il émet l'avis que les spermatozoïdes peuvent parcourir les $7 \mathrm{~cm}$ représentant la longueur des cornes utérines en $25 \mathrm{mn}$ environ, donc en moins de 2 heures. Pour PARKER, ses observations sont en accord avec celles faites antérieurement par Lim et CHAO (I926) qui obtiennent la gestation de lapines dont ils ont inversé un fragment de corne utérine. Chang et Pincus (I95 I) admettant que la vitesse de déplacement des spermatozoïdes est la même in vivo et in vitro, pensent aussi que leur transport peut être assuré grâce à leur motilité.

Tous les autres auteurs et ils sont les plus nombreux, attribuent à l'utérus un rôle actif dans le transit des spermatozoïdes.

Ainsi pour LEUCKARDT (I853) les spermatozoïdes de Lapins sont véhiculés par un antipéristaltisme des parois utérines, opinion partagée par MarTin (I895). Plus récemment Krehbiel et Cars'tens (I939) montrent qu'une stimulation de la vulve de Lapine est nécessaire pour obtenir le transport des particules inertes, du vagin dans le reste du tractus génital.

De nombreux auteurs ont directement étudié l'activité musculaire de l'utérus et des cornes utérines. WISJENBECK (I922), et WISJENBECK et Grevenstuck (I922) mettent en évidence un péristaltisme des parois 
utérines de la Lapine. Pendant l'œstrus, 1'utérus est très actif (BrouHA, Simonne'T, I927, chez la Rate), et au moment du coït, il se produit d'après WeStMANN (I926) une hyperhémie du tractus génital de la Lapine, avec arrêt des contractions, celles-ci réapparaissant ensuite avec une plus grande fréquence pour diminuer un peu avant l'ovulation de la Lapine (Westmann, I926 ; Rexnoldos et Friedman, I930; Sager et Leonard, 1936); chez la Rate (Clark, Knauss, Parkes, I925-I926), elles ne cessent qu'après l'ovulation. Chaque contraction utérine serait la continuation d'une contraction tubaire, se déplaçant le long des cornes puis s'atténuant dans la région du cervix (POMPEN, I933; WestManN, I929; REYNOLDS, I949).

Sur la Rate anesthésiée à 1'amytal de Sodium, Rossman (I937) met en évidence deux sortes de péristaltisme : l'un affectant un fragment de cornes de I cm, l'autre de plus petites fractions. Ces contractions produisent, au sein du liquide utérin, des courants rapides dirigés vers le sommet des cornes de l'utérus. Les spermatozoïdes de Rat véhiculés dans ce milieu utérin (HARTMAN et BALL, I930) remonteraient un peu plus lentement en l'absence de liquide (WARREN, I938), sa présence favorisant la fécondation, sans toutefois être indispensable (ĹtínARD, I950).

Van Demark et Moeller (I95) qui ont trouvé des montées de spermatozoïdes quasi-instantanées, chez la Vache, en ont cherché l'explication dans une activité du tractus femelle. Constatant des variations de pression intra-mammaire au moment de l'accouplement, HAXs et VAN DEMARK (I95I-I952) en concluent que la stimulation des organes sexuels provoque une décharge d'ocytocine. Van Demark et Hays (I952) enregistrant alors les contractions utérines qui déclenchent cette décharge d'ocytocine, en plaçant un ballonnet rempli d'eau, in vivo, dans l'utérus, constatent que la vue, l'approche d'un Taureau, le simulacre d'accouplement et le coït avec éjaculation, sont autant de stimuli qui déterminent une augmentation du tonus utérin et une apparition de contractions dans les minutes qui suivent. Normalement, il y a des contractions régulières, fréquentes et de faible amplitude pendant l'œstrus de la Vache, alors qu'au milieu du cycle œstral, elles sont de plus grande amplitude, mais moins fréquentes (HAYS, VAN DEMARK, I953). Opérant in vivo et in vitro, Hays et Van Demark (I953), et Van Demark et Hays (I95I) constatent que les contractions utérines peuvent être accentuées par l'ocytocine et atténuées par l'épinéphrine. Salisbury et VAN Demark (I952) en concluent que l'ascension rapide des spermatozoïdes dans l'appareil génital de la Vache est due à des contractions de l'utérus, résultant d'une excitation nerveuse qui détermine une sécrétion d'ocytocine agissant sur les muscles utérins. En injectant au moment du coït de l'ocytocine à des vaches, HAYs et VAN DEMARK (1953) constatent une augmentation significative du taux de conception par rapport aux témoins; malheureuse- 
ment, ils obtiennent la même augmentation avec l'injection d'épinéphrine. Rowson (I955) a montré, chez la Vache, que les contractions utérines déterminent une progression de substances opaques aux rayons $\mathrm{X}$, et fixé leur vitesse de progression $(75 \mathrm{mn}$ pour atteindre le sommet des trompes de Fallope), vitesse que l'on ne peut comparer à celle de cellules comme les spermatozoïdes.

\section{2o Recherches personnelles.}

Pour les raisons indiquées au sujet du cervix, des spermatozoïdes morts ont été utilisés dans l'étude de la montée des spermatozoïdes dans les cornes utérines. Pour éviter toute cause d'erreur, les gamètes mâles ont été séparés par centrifugation du liquide spermatique, la seule pesanteur risquant de déterminer un écoulement $\mathrm{du}$ sperme le long du tractus, au cours des manipulations. Les spermatozoïdes ont été ensuite tués par le froid pour préserver des substances pouvant éventuellement avoir une action sur l'utérus.

a) Étude in vivo. - Dans une première expérience, des spermatozoïdes morts sont déposés dans la partie proximale, c'est-à-dire cervicale, des cornes utérines. La lumière du col de l'utérus présentant une série de chicanes dues à la présence de nombreux replis de la paroi, il est impossible, à la différence de la Vache, de déposer par voie cervicale, des spermatozoïdes dans l'utérus de la Brebis. Nous avons donc été conduits à opérer par laparatomie de Brebis anesthésiées.

Deux anesthésiques ont été utilisés : le Nesdonal et le chloral, ce dernier n'affectant pas le rythme des contractions des fragments d'utérus de Brebis anesthésiées (Ambache, Hammond, I949). I1 n'est pas question, en effet, d'avoir recours à l'éther ou à d'autres anesthésiques administrés par voie gazeuse, qui, par le goût désagréable communiqué à la viande, la rendent impropre à la consommation.

Avec le Nesdonal (Thibault, Ortavant, LAplaud, I948), on fait une injection lente en intraveineuse, dans la veine jugulaire, d'une solution à ro $\%$ de nesdonal et ro \% de glucose. Une quantité de 5 à ro cc suffit pour provoquer rapidement le sommeil. L'anesthésie est maintenue en injectant en goutte à goutte une solution à I \% de nesdonal et Io \% de glucose.

Le chloral inoculé en injection intraveineuse sous forme d'une solution à $20 \%$ d'hydrate de chloral et $2 \%$ de citrate de sodium, provoque le sommeil plus lentement que le nesdonal. La narcose est maintenue par injection goutte à goutte de la même solution de chloral.

L'animal anesthésié est maintenu sur le dos, les pattes de derrière fixées par deux cordes qui coulissent dans deux poulies fixées au plafond. Après avoir pratiqué une ouverture de 6 à Io $\mathrm{cm}$ sur la ligne blanche, 
le train postérieur est levé, les épaules et la tête restant seules sur la table d'opération. Dans cette position, les viscères retombent, par leur propre poids, dégageant la partie postérieure de l'abdomen, done le tractus génital qui est facilement accessible. La position de l'animal ne gêne guère la respiration, mais elle provoque fréquemment des vomissements qui peuvent entraîner la mort par obstruction de la trachée, si l'on ne prend soin de placer une sonde intratrachéale débouchant largement à l'extérieur.

En surveillant le rythme respiratoire et en réglant convenablement le débit du goutte à goutte, les syncopes sont rares et on obtient une narcose permettant facilement 1'opération qui dure normalement I5 à zo minutes, comprenant le dépôt des spermatozoïdes morts au bas des cornes utérines au moyen d'une aiguille munie d'un mandrin, et la suture de la paroi médio-ventrale de l'abdomen, puis de la peau. L'anesthésie peut être prolongée si des observations ou des prélèvements doivent être effectués.

L'animal se réveille généralement 30 minutes à $\mathrm{I}$ heure plus tard, la fin de la narcose apparaissant plus vite après l'anesthésie au nesdonal.

Les Brebis, accouplées avant et après l'opération avec un Bélier vasectomisé, sont abattues 4 heures plus tard (1'anesthésique ayant, dans ces conditions, cessé d'agir depuis un certain temps).

La recherche des spermatozoïdes par la technique des frottis montre (tableau XVI) que les gamètes mâles sont dans tous les cas, et quel que soit l'anesthésique utilisé, présents tout au long des cornes utérines, le sommet étant toujours atteint. Les gamètes ont donc progressé par suite d'une intervention de l'utérus.

\section{TABLEAU XVI}

\begin{tabular}{|c|c|c|c|c|}
\hline \multirow{2}{*}{ Anesthésique } & \multirow{2}{*}{$\begin{array}{c}\text { Numéro } \\
\text { Brebis }\end{array}$} & \multicolumn{3}{|c|}{ Nombre de spermatozoïdes } \\
\hline & & Bas des Cornes & Milieu des Cornes & Sommet des Cornes \\
\hline $\begin{array}{l}\text { Nesdonal } \ldots \ldots \ldots \\
\text { Nesdonal } \ldots \ldots \ldots \\
\text { Nesdonal } \ldots \ldots\end{array}$ & $\begin{array}{r}291 \\
16 \\
+84\end{array}$ & $\begin{array}{l}\infty \\
\infty \\
\infty\end{array}$ & $\begin{array}{r}49 \\
35 \mathrm{I} \\
\infty\end{array}$ & $\begin{array}{c}8 \\
10 \\
\infty\end{array}$ \\
\hline $\begin{array}{l}\text { Chloral............ } \\
\text { Chloral .......... } \\
\text { Chloral........ }\end{array}$ & $\begin{array}{r}12 \\
243 \\
9\end{array}$ & $\begin{array}{l}\infty \\
\infty \\
\infty\end{array}$ & $\begin{array}{l}\infty \\
\infty \\
\infty\end{array}$ & $\begin{array}{r}2 \mathrm{I} \\
32 \\
3\end{array}$ \\
\hline
\end{tabular}

La même expérience a été réalisée sur des Brebis en milieu de diœestrus, en utilisant seulement le chloral (tableau XVII).

Une remontée très faible de spermatozoïdes s'est produite, ceux-ci ayant atteint le milieu des cornes dans 3 cas sur 6 et n'étant parvenus au sommet que dan un seul cas, et en très petit nombre. 
Ces résultats montrent donc que la progression des spermatozoïdes morts dépendrait d'une activité de l'utérus, activité qui serait plus faible en milieu de diøestrus qu'en oestrus.

TABLEAU XIII

\begin{tabular}{|c|c|c|c|c|c|}
\hline \multirow{2}{*}{ Anesthésique } & \multirow{2}{*}{$\begin{array}{l}\text { Numéro } \\
\text { Brebis }\end{array}$} & \multirow{2}{*}{ Côté } & \multicolumn{3}{|c|}{ Nombre de spermatozoïdes } \\
\hline & & & Bas des Cornes & $\begin{array}{c}\text { Milieu } \\
\text { des Cornes }\end{array}$ & $\begin{array}{c}\text { Sommet } \\
\text { des Cornes }\end{array}$ \\
\hline Chloral ..... & 40 & $\begin{array}{l}\mathrm{G} \\
\mathrm{D}\end{array}$ & $\begin{array}{l}\infty \\
\infty\end{array}$ & $\begin{array}{r}0 \\
29\end{array}$ & $\begin{array}{l}0 \\
0\end{array}$ \\
\hline Chloral .. & $3 \mathrm{I}$ & $\begin{array}{l}\mathrm{G} \\
\mathrm{D}\end{array}$ & $\infty$ & 0 & $\begin{array}{l}\circ \\
\circ\end{array}$ \\
\hline Chloral ... & 566 & $\begin{array}{l}\mathrm{G} \\
\mathrm{D}\end{array}$ & $\begin{array}{l}\infty \\
\infty\end{array}$ & $\begin{array}{l}\mathrm{I} 54 \\
\mathrm{I} 35\end{array}$ & $\begin{array}{l}4 \\
0\end{array}$ \\
\hline
\end{tabular}

b) Etude in vitro. - - Une étude in vitro a d'ailleurs permis de confirmer ces résultats.

Des tractus génitaux de 6 brebis, préalablement accouplées avec un bélier vasectomisé, sont mis en survie. Le prélèvement de l'organe est fait, aussitôt l'animal tué, le tractus étant sectionné au niveau de la partie cervicale. Pour chaque expérience, nous avons utilisé les deux cornes utérines. Chaque moitié supérieure du tractus est placée dans un cylindre qui communique avec un serpentin lui-même relié à un récipient contenant $\mathrm{du}$ Tyrode. Une deuxième tubulure $\mathrm{du}$ cylindre permet la vidange totale, un courant d'oxygène alimentant le milieu de survie par une troisième tubulure. Les deux cylindres et le serpentin, qui depuis le flacon de tyrode sert à les alimenter, baignent dans l'eau d'une cuve à thermostat, à la température de $3^{8^{\circ}} 5$. La partie proximale du tractus, où sont déposés les spermatozoïdes, est ensuite ligaturée et fixée à 1'extrémité d'une baguette de verre solidaire du bouchon qui obture la partie inférieure du cylindre. La trompe de Fallope ligaturée est libre, de telle sorte que le tractus puisse se contracter librement. Les opérations sont faites avec le maximum de soins et le minimum de manipulations pour éviter tout déplacement accidentel des spermatozoïdes.

I a composition de la solution de Tyrode est la suivante :

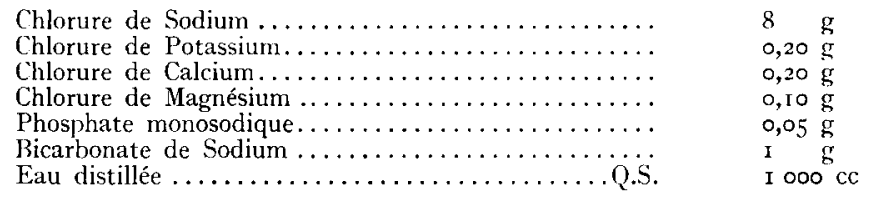

4 heures après la mise en place des organes dans la cuve à survie, ceux-ci sont prélevés, les trompes étant toujours maintenues à un niveau plus élevé que les cornes utérines, pour éviter toute erreur consécutive à une intervention de la pesanteur. 
De la lecture des frottis, il ressort (tableau XVIII), que les résultats obtenus in vitro confirment ceux réalisés in vivo. Alors que dans les tractus génitaux de Brebis en cestrus, les spermatozoïdes sont retrouvés au sommet des cornes, ceux-ci ne sont jamais présents à la partie supérieure des cornes utérines de Brebis en dicstrus, la partie moyenne n'étant même atteinte que dans deux cas sur six.

c) Interprétation des résultats. - Ces résultats montrent donc que l'utérus joue un rôle dans le transit des spermatozoïdes du cervix à la jonction utéro-tubaire, les cornes utérines se comportant différemment pendant l'cestrus et le milieu du dicestrus.

Nous avons donc été conduits à étudier les contractions des cornes utérines à différents moments du cycle ostral. GunN (I944), Ambache et HAmmond (I948), se sont, en effet, seulement attachés à étudier l'action de différentes substances sur l'utérus de Brebis gestantes ou en anœstrus.

TABLEAU XVIII

\begin{tabular}{|c|c|c|c|c|c|}
\hline \multirow{2}{*}{$\begin{array}{c}\text { Moment } \\
\text { du cycle sexuel }\end{array}$} & \multirow[b]{2}{*}{ Numéro brebis } & \multicolumn{3}{|c|}{ Nombre de spermatozoïdes } & \multirow[b]{2}{*}{ Côté } \\
\hline & & Bas des cornes & $\begin{array}{l}\text { Milieu } \\
\text { des cornes }\end{array}$ & $\begin{array}{l}\text { Sommet } \\
\text { des cornes }\end{array}$ & \\
\hline \multirow[t]{2}{*}{ Estrus $\ldots \ldots \ldots$} & $r_{3} \mathrm{I}$ & $\infty$ & $\infty$ & 612 & G \\
\hline & & $\infty$ & $\infty$ & 2216 & $\mathrm{D}$ \\
\hline \multirow[t]{2}{*}{ Estrus } & $17 \mathrm{I}$ & $\infty$ & 245 & I & G \\
\hline & & $\infty$ & $x$ & 72 & $\mathrm{D}$ \\
\hline \multirow[t]{2}{*}{ (Estrus } & 494 & $\infty$ & tos & 44 & $\mathrm{G}$ \\
\hline & & $\propto$ & $\approx$ & 240 & $\mathrm{D}$ \\
\hline Hilieu $\ldots \ldots \ldots \ldots$ & I 39 & $\infty$ & 0 & 0 & $G$ \\
\hline Dioestrus . . . . . . & & $\infty$ & 0 & 0 & $\mathrm{D}$ \\
\hline Milieu . . & 45 & $\infty$ & c & o & $\mathrm{G}$ \\
\hline Diœestrus & & $\infty$ & 300 & 0 & D \\
\hline Vilieu ..... & $I_{32}$ & $\infty$ & 0 & $\circ$ & $\mathrm{G}$ \\
\hline Diœstrus ... & & $\infty$ & 6 & 0 & $\mathrm{D}$ \\
\hline
\end{tabular}

Nous avons délaissé la technique du ballonnet utilisé par VAN DEMARK et Hays (I952) qui a le désavantage de distendre anormalement les cornes utérines. REYNoLDS (I949) signale les inconvénients de cette distension qui détermine une plus grande augmentation du volume intrautérin que de la surface des cornes. Il en résulte qu'une faible contraction du myomètre d'un utérus distendu peut aroir un effet apparent plus grand qu'une très forte contraction d'un utérus moins distendu. L'opération, à pression et volume constants, ne crée pas en outre, des conditions expérimentales comparables, car on ne tient pas compte des variations de l'état physiologique de l'utérus. Dans certains cas, enfin, la distension de 1'utérus peut même augmenter la contractilité du myomètre de façon réelle ou apparente (REYNOLDS, I949).

Dans ces conditions, les contractions des cornes utérines, mises en survie, in vitro, sont enregistrées de la manière suivante : la région du 
tractus génital correspondant à la jonction utéro-tubaire est reliée par un fil, dont la tension est toujours la même, à un bras de levier portant un stylet dont les déplacements sont enregistrés sur un cylindre noirci.

A partit d'un même animal, on peut donc réaliser deux enregistrements, un sur chaque corne utérine.

On constate (graphique I) que les cornes utérines de Brebis en œstrus, présentent des contractions, au rythme de 2 à 3 par minute, des contractions de plus grande amplitude étant séparées par des plus petites.

En diœstrus, le rythme des contractions est sensiblement le même mais celles-ci sont beaucoup plus atténuées et de faible amplitude (graphique 2).

Cette différence d'importance des contractions utérines permet donc de penser que celles-ci, responsables du transport des spermatozoïdes morts du cervix à la jonction utéro-tubaire, assurent leur progression plus rapidement pendant l'œestrus qu'en milieu de diœstrus.

Evans et Miller (I936), Cupps et Asdell, (I944) chez la Vache, KING (1927) chez la Truie, constatent aussi que 1'activité de l'utérus, plus grande au moment de l'xestrus, diminue pour devenir faible, quelques jours après. HAYs et VAX DEMARK (I953) observent chez la Vache des petites contractions qui se produisent avec une assez haute fréquence pendant l'œstrus, tandis qu'en dicestrus ce sont des contractions de plus grande amplitude, mais plus espacées qui ont lieu. Pour ces auteurs, cependant, la progression des spermatozoïdes dépendrait d'une activité de l'utérus, due à une action de l'ocytocine sécrétée à la suite d'une excitation liée au coït. Si nous arons constaté comme ces auteurs que l'ocytocine provoque une augmentation de tonus de l'utérus en survie in vitro, aucune différence dans 1'amplitude et la fréquence des contractions des cornes utérines de Brebis n'est observable in vitro, que les animaux aient été ou non accouplés avec un Bélier vasectomisé. La réaction de l'utérus à l'ocytocine in wiro cessant I à 3 minutes après le coït (VAN DEMARK et HAYS, I952), il faut admettre comme ces auteurs, que les cornes utérines interviennent dans le transport des gamètes dans les 3 minutes qui suivent l'accouplement. Or, nous avons constaté non seulement que la montée des spermatozoïdes dans le tractus génital de la Brebis nécessite plusieurs heures, mais encore que le cervix n'est traversé qu'après $I_{5}$ ou 30 minutes et par la seule motilité des spermatozoïdes. L'action directe de l'utérus mise en évidence ne peut donc se manifester chez la Brebis qu'une fois le cervix franchi par les spermatozoïdes.

Cette fonction des cornes utérines est d'ailleurs mise expérimentalement en évidence in vitro, par inhibition des contractions par le Nesdonal. A cet effet, une des cornes utérines d'un tractus génital sert à l'enregistrement des contractions, tandis que des spermatozoïdes morts 


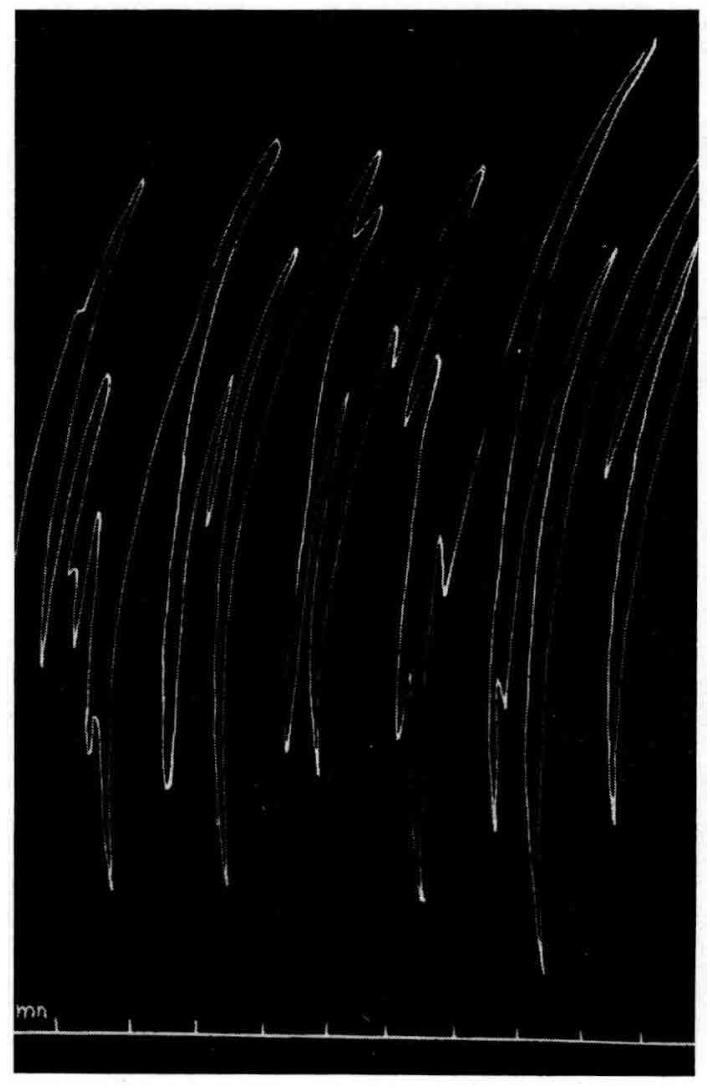

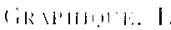

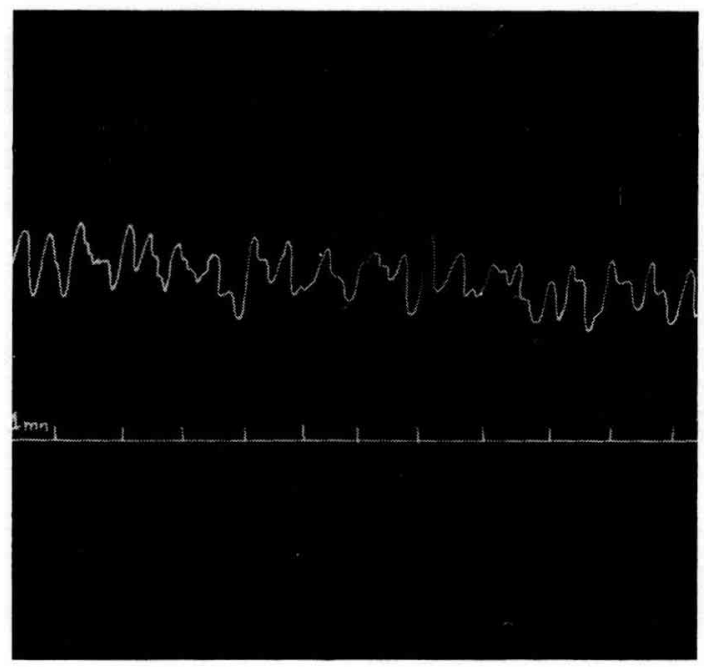

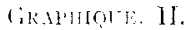



sont déposés an bas de la $2^{\mathrm{e}}$ corne, l'inhibition des contractions étant suivie sur la première (graphique III).

En fin d'expérience, une élimination du Nesdonal par lavage fait réapparaître les contractions de la corne qui est donc toujours en survie (graphique IV).

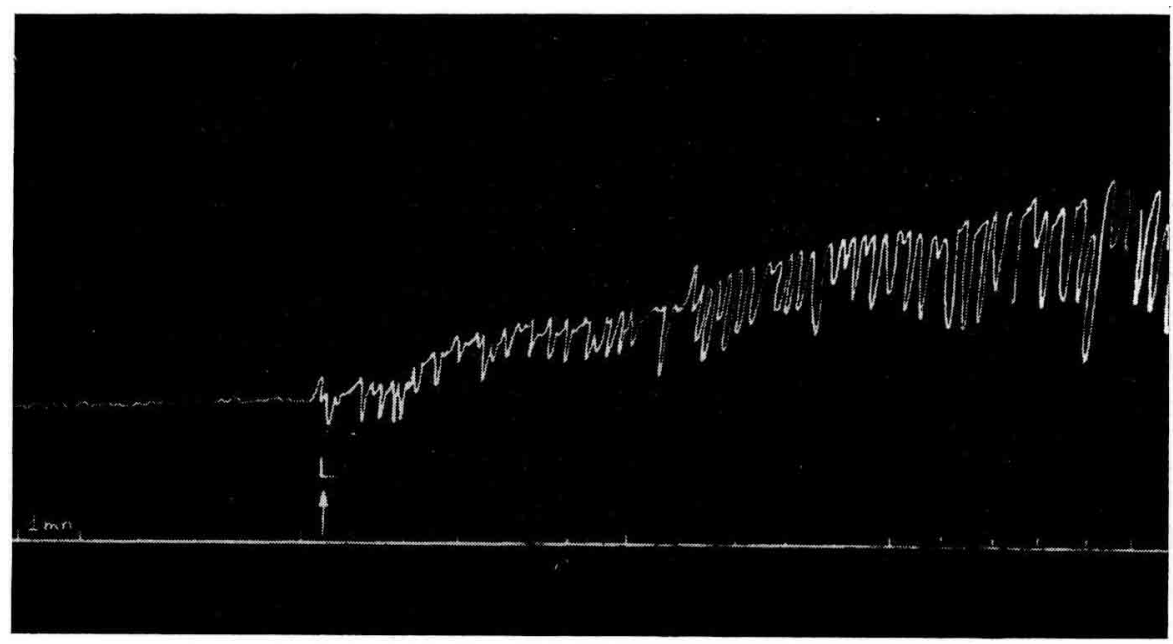

Graphique 1 V. - I $=$ lavage.

On constate (tableau XIX) qu'il ne se produit aucune progression des spermatozoïdes morts dans les cornes utérines de Brebis dont les contractions sont ainsi inhibées.

\section{TABLEAU XIX}

\begin{tabular}{|c|c|c|c|}
\hline \multirow{2}{*}{$\begin{array}{l}\text { Nurnéro } \\
\text { Brebis }\end{array}$} & \multicolumn{3}{|c|}{ Nombre de spermatozoïdes } \\
\hline & $\begin{array}{c}\text { Base des cornes } \\
\text { utérines }\end{array}$ & $\begin{array}{c}\text { Milieu des cornes } \\
\text { utérines }\end{array}$ & $\begin{array}{c}\text { Sommet des cornes } \\
\text { utérines }\end{array}$ \\
\hline т 08 & $\infty$ & $\circ$ & $\circ$ \\
\hline So & $\infty$ & I & 0 \\
\hline $\mathrm{s}_{2}$ & $\infty$ & 0 & o \\
\hline 77 & $\infty$ & $\circ$ & $\circ$ \\
\hline
\end{tabular}

La seule intervention des contractions utérines de la Brebis peut donc expliquer le transport des spermatozoïdes de Bélier dans cette partie du tractus femelle. Ces contractions déterminent un brassage du contenu utérin, puisqu'en déposant des spermatozoïdes morts débarrassés de liquide spermatique au sommet des cornes de brebis en œstrus, in vivo et in vitro, ceux-ci sont retrouvés au bas des mêmes organes 4 heures après leur dépôt (tableau XX). 
TABLEAU XX

\begin{tabular}{|c|c|c|c|c|}
\hline \multirow{2}{*}{ Technique } & \multirow{2}{*}{$\begin{array}{l}\text { Numéro } \\
\text { Brebis }\end{array}$} & \multicolumn{3}{|c|}{ Nombre de spermatozoïdes } \\
\hline & & Bas des cornes & Milieu des cornes & Sommet des comes \\
\hline "in vivo" & $\begin{array}{l}536 \\
834\end{array}$ & $\begin{array}{r}531 \\
49\end{array}$ & $\begin{array}{c}\infty \\
47^{6}\end{array}$ & $\begin{array}{l}\infty \\
\infty\end{array}$ \\
\hline "in vitro" & $\begin{array}{l}972 \\
616\end{array}$ & $\begin{array}{r}15 \\
689\end{array}$ & $\begin{array}{l}218 \\
\infty\end{array}$ & $\begin{array}{l}\infty \\
\infty\end{array}$ \\
\hline
\end{tabular}

Ces résultats confirment ceux obtenus par PARKER (I93I) avec des spermatozoïdes vivants déposés à la partie supérieure des cornes utérines de lapines; mais les arguments de cet auteur, attribuant à la seule motilité des spermatozoïdes leur déplacement, sont vraisemblablement erronés, les contractions de l'utérus jouant un rôle capital dans ce phénomène constaté tout au moins chez la Brebis avec des spermatozoïdes morts.

Toutefois, les contractions utérines ne peuvent suffire à expliquer la progression des spermatozoïdes dans cette zone du tractus génital femelle, puisqu'il n'y a pas, en opérant avec des spermatozoïdes morts (tableaux XVI et XVIII), l'accumulation des gamètes mâles (fig. 4), mise en évidence avec les spermatozoïdes vivants dans l'étude sur leur vitesse de remontée.

On peut donc affirmer que si les contractions utérines suffisent à expliquer une progression des spermatozoïdes dans les cornes utérines de Brebis en ostrus, cette progression est également pour une certaine part sous la dépendance de la motilité propre des gamètes mâles.

\section{III. - FACTEURS QUI CONDITIONNENT LA PROGRESSION DES SPERMATOZOIDES A U NIVEA U DE LA JONCTION UTÉRO TUBAIRE}

\section{Résultats antérieurs.}

Il est curieux de constater que le mécanisme physiologique du transit des spermatozoïdes au niveau de la jonction utéro-tubaire, ait fait l'objet de peu de travaux. Cette fraction très brève, du tractus génital femelle, joue pourtant un rôle aussi important que le cervix dans la montée des gamètes mâles. Une très faible partie des spermatozoïdes accumulés, au sommet des cornes utérines, franchissent en effet cet étranglement, pour continuer leur progression dans les trompes de F'allope.

Ce sont surtout des études anatomiques de cette région qui ont été faites. KELL (I928) indique qu'il y a, au niveau de la jonction utérotubaire, des replis muqueux, variables, en nombre et en dimensions, la corne utérine pouvant être considérée comme un prolongement direct de l'oviducte, à la façon du " pavillon d'une trompette ». I EE (I928), 
outre ces replis, signale chez un certain nombre d'espèces (Cobaye, Souris, Rate, I_apine...) la présence de couches musculaires circulaires suggérant l'existence d'un sphincter, une structure rappelant une valve interrompant la continuité entre les trompes et les cornes utérines (ANDERSEN, I928) ; c'est ce que Lí́onard, PerLman (I949) appellent chez la Rate, la valve utéro-tubaire. Des études physiologiques ont visé à déterminer quantitativement les pressions nécessaires pour faire passer un fluide de l'utérus dans l'oviducte, le franchissement, en sens inverse, étant aisé. Les auteurs opèrent soit sur l'animal anesthésié, soit sur des organes prélevés après abattage.

Alors que PARKer (I93I) prétend que le passage de particules inertes de l'utérus dans les trompes de Fallope est aisément obtenu chez la Lapine, REYNoLDS (I930) opérant par fistule utérine de Lapines non anesthésiées, ne force la " jonction utéro-tubaire " qu'avec des pressions de Io à $200 \mathrm{~mm}$ de mercure. ANDERsen (I928) admet qu'il faut des pressions relativement faibles pour faire passer des fluides dans l'oviducte de la Vache ou de la Brebis. Alden (I943) chez la Rate, n'obtient ces résultats qu'avec des pressions plus élevées $(5 \mathrm{I} \mathrm{mm}$ de mercure pendant le précestrus et le dicestrus) l'état de contraction de la jonction utérotubaire étant maximum pendant l'œstrus de la Rate (Ioo mm de mercure). LÉonard et Perliman (I949) sur la Rate, à l'inverse de Parker (I93I) sur la Lapine, n'obtiennent pas la progression de particules inertes dans cet étranglement, et confirment le point de vue d'ALDEN (I943), la valve utéro-tubaire permet le passage des spermatozoïdes vivants seulement pendant l'œstrus et de I 2 heures à 72 heures après. Pour LÉONARD et PerL,man (r949), la motilité des spermatozoïdes de Rat est donc nécessaire au franchissement de cette zone. Une substance stimulerait localement une relaxation de la valve qui aurait une action sélective, puisque en mélangeant des spermatozoïdes étrangers à ceux du Rat, seuls, ceux-ci passent. En fait, on comprend mal cette sélectivité de la jonction utérotubaire, et il semble bien plus logique d'admettre que le comportement des spermatozoïdes étrangers, et en particulier, leur métabolisme, est différent de celui des spermatozoïdes de Rat. En effet, opérant sur la Brebis, PHILlips et ANDREws (1937) rapportent au contraire que sur 3 Brebis inséminées (sur 7) ils obtiennent ell mélangeant des spermes de bélier et de rat, une remontée des spermatozoïdes des deux espèces, quoiqu'un peu moins rapide pour ceux de rat. Or, ces auteurs, contrairement à nos résultats, observent des spermatozoïdes de bélier à la partie supérieure des trompes dès la $30^{\mathrm{e}}$ minute après le coït.

\section{$2^{\circ}$ Recherches personnelles.}

Des spermatozoïdes non séparés du liquide spermatique, mais tués par le froid, sont déposés, au travers de la paroi, au sommet des cornes 
utérines de Brebis en œestrus, accouplées ou non avec un Bélier vasectomisé, anesthésiées au chloral et abattues 5 heures après le début de 1'opération.

La recherche de spermatozoïdes par la technique des frottis a été abandonnée, la partie extérieure des trompes étant récupérée par raclage, comme le contenu tubaire. Dans ces conditions, les gamètes mâles qui peuvent être déposés accidentellement sur les oviductes au cours de l'opération, faussent les résultats. La réalisation de lames témoins par frottis de la paroi externe des fragments de trompes avant la récupération du milieu utéro-tubaire sur une deuxième lame, n'a pas amélioré les résultats. Devant 1'incohérence de ceux-ci, la technique de perfusion a été utilisée.

La recherche des spermatozoïdes à différents niveaux des trompes de Iiallope montre que les spermatozoïdes morts ne franchissent jamais la jonction utéro-tubaire. Aucun gamète n'est trouvé à un niveau supérieur à celui du dépôt (tableau XXI).

La même étude réalisée in vitro, sur des tractus génitaux en survie dans du 'Tyrode oxygéné à $3^{8}, 5^{\circ} \mathrm{C}$ aboutit aux mêmes résultats : aucune progression des spermatozoïdes morts ne se produit (tableau XXI).

\section{TABLEAL XXI}

\begin{tabular}{|c|c|c|c|c|c|c|}
\hline Technique & $\begin{array}{c}\text { Numéro } \\
\text { Brebis }\end{array}$ & $\begin{array}{l}\text { Sommet des } \\
\text { Cornes }\end{array}$ & $\begin{array}{l}3 / 5 \text { médian } \\
\text { Isthme }\end{array}$ & $\begin{array}{l}\text { Haut Isthme } \\
\text { Bas Ampoule }\end{array}$ & $\begin{array}{l}\text { Haut } \\
\text { Ampoule }\end{array}$ & Côté \\
\hline \multirow{3}{*}{ Anesthésie Chloral } & $5^{1} 3$ & $\begin{array}{l}\propto \\
\propto\end{array}$ & $\begin{array}{l}0 \\
0\end{array}$ & $\begin{array}{l}0 \\
0\end{array}$ & $\begin{array}{l}0 \\
0\end{array}$ & $\begin{array}{l}\mathrm{G} \\
\mathrm{D}\end{array}$ \\
\hline & 839 & $x$ & o & $\circ$ & $\circ$ & $\mathrm{G}$ \\
\hline & $4 \mathrm{I} 4$ & $\infty$ & $\begin{array}{l}0 \\
0\end{array}$ & $\begin{array}{l}0 \\
0\end{array}$ & $\begin{array}{l}0 \\
0\end{array}$ & $\begin{array}{l}G \\
D\end{array}$ \\
\hline \multirow{3}{*}{ Survie in vitro } & 182 & $\begin{array}{l}x \\
x\end{array}$ & $\begin{array}{l}0 \\
0\end{array}$ & $\begin{array}{l}0 \\
0\end{array}$ & $\begin{array}{l}0 \\
0\end{array}$ & $\begin{array}{l}\mathrm{G} \\
\mathrm{D}\end{array}$ \\
\hline & 318 & $\infty$ & $\circ$ & 0 & 0 & $\stackrel{G}{D}$ \\
\hline & 195 & $\begin{array}{l}\infty \\
x\end{array}$ & $\begin{array}{l}0 \\
0\end{array}$ & $\begin{array}{l}0 \\
0\end{array}$ & $\begin{array}{l}0 \\
0\end{array}$ & $\begin{array}{l}\mathrm{G} \\
\mathrm{D}\end{array}$ \\
\hline
\end{tabular}

L'anesthésie, ou la surrie, du tractus génital risquant d'avoir une action sur la perméabilité aux spermatozoïdes de la jonction utéro-tubaire, des expériences ont été reprises, in vivo et in vitro, en déposant au sommet des cornes utérines des spermatozoïdes très motiles, fraîchement éjaculés.

I,es spermatozoïdes sont alors retrouvés dans la partie supérieure des trompes de Fallope de brebis anesthésiées, et dans l'isthme et la partie inférieure de l'ampoule ( 2 oviductes sur 4) de tractus génitaux mis en survie (tableau XXII). 
TABLEAU XXII

\begin{tabular}{|c|c|c|c|c|c|c|}
\hline $\begin{array}{l}\text { Numéro } \\
\text { Brebis }\end{array}$ & Technique & Côté & $\begin{array}{c}\text { Sommet des } \\
\text { Cornes }\end{array}$ & $\begin{array}{l}3 / 5 \text { médian } \\
\text { Isthme }\end{array}$ & $\begin{array}{l}\text { Haut Isthme } \\
\text { I/2 inf. Amp. }\end{array}$ & $\begin{array}{l}\text { I } / 2 \text { sup. } \\
\text { Ampoule }\end{array}$ \\
\hline$I 69$ & Anesthésie Chloral & $\begin{array}{l}G \\
D\end{array}$ & $\begin{array}{l}\infty \\
\infty\end{array}$ & $\begin{array}{c}\infty \\
1.39^{2}\end{array}$ & $\begin{array}{l}22 \\
28\end{array}$ & $\begin{array}{l}6 \\
0\end{array}$ \\
\hline I 58 & Anesthésic (hloral: & $\begin{array}{l}\mathrm{C} \\
\mathrm{D}\end{array}$ & $\begin{array}{l}\infty \\
\infty\end{array}$ & $\begin{array}{l}1.44 \mathrm{I} \\
2.16_{3}\end{array}$ & $\begin{array}{r}6 \\
1.1+9\end{array}$ & $\begin{array}{l}19 \\
25\end{array}$ \\
\hline $\mathrm{I}+\mathrm{I}$ & Anesthésie Chloral & $\begin{array}{c}\mathrm{G} \\
\mathrm{D}\end{array}$ & $\begin{array}{l}\infty \\
\infty\end{array}$ & $\begin{array}{l}2.7 \mathrm{II} \\
\mathrm{I} .218\end{array}$ & $\begin{array}{r}2 \\
13+4\end{array}$ & $\begin{array}{l}0 \\
\mathbf{I}\end{array}$ \\
\hline 513 & Survie in vitro & $\frac{G}{D}$ & $\begin{array}{l}\infty \\
\infty\end{array}$ & $\begin{array}{r}18 \\
256\end{array}$ & $\begin{array}{l}6 \\
0\end{array}$ & $\begin{array}{l}0 \\
0\end{array}$ \\
\hline 830 & Survie in vitro & $\begin{array}{l}\mathrm{G} \\
\mathrm{D}\end{array}$ & $\begin{array}{l}\infty \\
\infty\end{array}$ & $\begin{array}{r}525 \\
40\end{array}$ & $\begin{array}{l}3 \\
0\end{array}$ & $\begin{array}{l}\circ \\
0\end{array}$ \\
\hline
\end{tabular}

La perméabilité aux spermatozoïdes vivants de la jonction utérotubaire de Brebis en œstrus existe donc, que l'on opère sur l'animal anesthésié ou sur l'appareil génital en survie, et son franchissement par les gamètes mâles ne peut être imputé qu'à leur propre motilité.

\section{IV. - FACTEURS QUI CONDITIONNENT LA PROGRESSION DES SPERMATOZOIDES DANS LES TROMPES DE FALLOPE}

\section{Résultats antérieurs.}

Les opinions les plus anciennement émises sur le mécanisme de progression des spermatozoïdes dans les trompes de Fallope attribuent un rôle important aux contractions tubaires et plus particulièrement aux mouvements antipéristaltiques (BISCHOFF, I842; LEUCKARDT, I853; LUDWIG, I86I). MimuRa (I94I) opérant sur des poules anesthésiées à l'évipan sodique, constate que les spermatozoïdes de coq progressent plus rapidement in vivo que dans un milieu liquide, et en conclut qu'une intervention de l'oviducte est nécessaire pour expliquer cette différence de vitesse de progression. Étudiant le rôle des différents facteurs possibles mis en jeu (contractions des parois, attraction capillaire, action ciliaire, pression mutuelle des viscères), il constate que des particules inertes (Bleu de Méthylène, carbone) progressent également vers l'ovaire dans les régions présentant, ou non, des mouvements ciliaires. Mrmura (I94I) en déduit que les contractions des parois musculaires de l'oviducte de la poule, notamment les mouvements antipéristaltiques, doivent être considérés comme le principal facteur assurant la remontée des gamètes mâles.

Chez d'autres espèces, des contractions tubaires ont d'ailleurs été mises en évidence; Wess'man (I926) signale une activité musculaire des trompes de Fallope, de Lapine, maxima pendant l'œstrus, quand il y a des follicules mûrs. De même, Seckinger (I923) décrit in vitro des 
contractions rapides et petites de segments d'oviductes, de Truie en cestrus, contractions qui disparaissent après l'arrivée des œufs dans l'utérus. Wislocki et GutTMACHER (I924) confirment ces résultats et montrent l'existence de variations cycliques des contractions tubaires chez la Truie, les plus accentuées se situant pendant l'œstrus. De I a FUENTE (I95I) tend à mettre en évidence une prédominance des mouvements péristaltiques dans l'isthme de la Fiemme, alors que l'ampoule est plutôt le siège de mouvements pendulaires.

Par contre, certains auteurs pensent que les spermatozoïdes progressent, en remontant contre un courant dirigé vers l'utérus et dû aux mouvements ciliaires, grâce à leur propriété rhéotactique (LOTT, I872; Adolphi, igo5; Hoehne, igi ; Felddman, I920). Pour Yamane et ITo (I932) la rhéotaxie des spermatozoïdes d'Étalon n'est pas un phénomène d'orientation; un contre-courant stimule les spermatozoïdes dont l'activité augmente. I'orientation des spermatozoïdes contre un courant et la rhéotaxie n'ont cependant d'après WALTON (I952) qu'un rôle hypothétique dans la progression des spermatozoïdes. PARKER (I93I) prétend, lui, avoir observé chez la Tortue et la Pigeonne des mouvements ciliaires $a b$ ovarian et une "bande discrète " de cils battant vers l'ovaire qu'il ne peut mettre en évidence chez la Lapine. D'après PARKER, les mouvements tubaires détermineraient l'existence de compartiments successifs et provisoires dans les trompes; sur les bords existerait un courant dirigé vers l'utérus et au centre un contre-courant vers l'ovaire, qui déterminerait une progression des spermatozoïdes, avec 1'aide de la pression des organes adjacents. PARKER (I93I) signale en effet, contrairement à Low (IgO2) et DewiTz (IgO3) que les spermatozoïdes ne sont ni attirés, ni en contact avec la paroi tubaire.

\section{Résultats personnels.}

Dans une première expérience des spermatozoïdes tués par le froid, débarrassés du liquide spermatique, sont déposés au bas des trompes de Fallope de Brebis en cstrus, en opérant, soit in vivo, après anesthésie au chloral, soit in vitro, dans une cuve à survie. Le lieu de mise en place des spermatozoïdes déposés en très grand nombre (de l'ordre du million), est soigneusement déterminé par un point lâche de fil de soie autour de la trompe. In vivo, l'aiguille est introduite dans la paroi utérine, près du sommet des cornes et guidée au travers de la jonction utéro-tubaire, dans l'isthme où elle pénètre de I $\mathrm{cm}$ environ. Les spermatozoïdes sont expulsés par un mandrin, dont le bout, au moment du dépôt des spermatozoïdes, atteint juste l'amorce du biseau de l'aiguille. Les plus grandes précautions doivent être prises lors de la mise en place des gamètes mâles, pour en limiter, avec précision, le lieu de dépôt, Ainsi, on ne peut 
injecter du sperme avec une micropipette, les spermatozoïdes étant immédiatement disséminés le long de l'oviducte, comme nous l'ont montré des mises au point préalables.

Dans ces conditions (tableau XXIII), les spermatozoïdes morts sont retrouvés 5 heures après leur dépôt dans la partie supérieure de la plupart des isthmes utilisés, mais n'ont pas atteint l'ampoule, bien que le nombre introduit soit considérable.

Tableau XXIII

\begin{tabular}{|c|c|c|c|c|c|}
\hline Technique & $\begin{array}{l}\text { Numéro } \\
\text { Brebis }\end{array}$ & Côté & $\begin{array}{l}1.2 \text { supérieure } \\
\text { Isthme }\end{array}$ & $\begin{array}{l}1 / 2 \text { inférieure } \\
\text { Ampoule }\end{array}$ & $\begin{array}{l}1 / 2 \text { supérieure } \\
\text { Ampoule }\end{array}$ \\
\hline \multirow{8}{*}{ Survie in vitro } & \multirow[t]{2}{*}{47} & (; & $\circ$ & 0 & 0 \\
\hline & & D & 0 & 0 & 0 \\
\hline & 44 & i & 448 & $\circ$ & 0 \\
\hline & \multirow{2}{*}{105} & G & $\begin{array}{c}D_{54} \\
\infty\end{array}$ & $\begin{array}{l}0 \\
0\end{array}$ & $\begin{array}{l}0 \\
0\end{array}$ \\
\hline & & D & 4 & 0 & 0 \\
\hline & \multirow[t]{2}{*}{195} & i & 317 & 0 & $\circ$ \\
\hline & & D & 82 & $\circ$ & $\circ$ \\
\hline & \multirow[t]{2}{*}{739} & (i & 44 & 0 & 0 \\
\hline & & D & 220 & 0 & 0 \\
\hline \multirow{9}{*}{ Anesthésie chloral } & 53 & G & 0 & 0 & 0 \\
\hline & \multirow{2}{*}{ I 29} & D & 0 & $\circ$ & $\circ$ \\
\hline & & D & $\begin{array}{l}0 \\
0 \\
0\end{array}$ & $\circ$ & 0 \\
\hline & \multirow{2}{*}{5} & (;) & $\begin{array}{l}0 \\
\infty\end{array}$ & $\begin{array}{l}0 \\
0\end{array}$ & $\begin{array}{l}0 \\
0\end{array}$ \\
\hline & & D & $\infty$ & 0 & 0 \\
\hline & \multirow[t]{2}{*}{6.35} & (i) & 452 & 0 & 0 \\
\hline & & D & $8 \mathrm{I} 4$ & 0 & 0 \\
\hline & \multirow[t]{2}{*}{$5^{12}$} & ir & 38 & 0 & 0 \\
\hline & & U & 75 & 0 & 0 \\
\hline
\end{tabular}

Il y a donc une progression des spermatozoïdes dans les oviductes de Brebis en oestrus, indépendante de la motilité des gamètes mâles et qui serait due aux contractions tubaires ou aux battements des cils de l'épithélium des trompes de I'allope. Pour déterminer la part de chacun de ces facteurs, dans une deuxième expérience, des spermatozoïdes morts sont déposés, selon la même technique, au bas de l'ampoule d'oviductes de Brebis en ostrus.

On constate que, dans ce cas, des spermatozoïdes sont retrouvés par perfusion, 5 heures plus tard, dans la partie inférieure de l'isthme, c'est-à-dire dans une zone plus éloignée de l'ovaire que leur lieu de dépôt. La progression des spermatozoïdes est donc indépendante du sens des mouvements ciliaires. KUO et IIM (I928) au contraire, ont montré qu'à la suite d'inversion de fragments d'oviductes de Lapines, la gestation est empêchée soit par arrêt de l'œuf, soit par une non-remontée des spermatozoïdes. Cette expérience a donc été reprise en inversant des fragments de $3 \mathrm{~cm}$ d'oviducte de Brebis. En opérant par laparatomie un segment d'oviducte est coupé, inversé et anastomosé, par 3 points de suture, aux extrémités opposées des sections laissées en place. Les 
Brebis accouplées avec un Bélier fécond, 6 mois après l'opération, pour que la cicatrisation des zones d'anastomose soit totale, sont abattues 5 à 6 jours après la fin des chaleurs. La recherche des spermatozoïdes est uniquement faite par examen des œufs, une remontée des spermatozoïdes étant admise quand l'étude histologique des œufs révèle leur fécondation. La perméabilité des trompes au niveau des zones de contact des segments inversés atteste de la continuité de la lumière tubaire.

\section{TaBleau XXIV}

Nombre de brebis dont les ceufs, ne franchissant pas la zone inversée, sont fécondés.

\begin{tabular}{l|c|c|c}
\hline \multicolumn{2}{|c|}{ Isthme } & \multicolumn{2}{|c|}{ Ampoule } \\
\hline $\begin{array}{l}\text { Témoin (section et sutu- } \\
\text { ration dans la même } \\
\text { position) } \\
2 \text { brebis sur 2 }\end{array}$ & Inversion & $\begin{array}{c}\text { Témoin (section et sutu- } \\
\text { ration dans la même } \\
\text { position) }\end{array}$ & Inversion \\
\hline
\end{tabular}

\section{TABLEAU XXV}

\begin{tabular}{|c|c|c|c|c|c|c|}
\hline \multirow{2}{*}{ Technique } & \multirow{2}{*}{$\begin{array}{l}\text { Spermatozoïdes } \\
\text { déposés }\end{array}$} & \multirow{2}{*}{$\begin{array}{l}\text { Numéro } \\
\text { Brebis }\end{array}$} & \multicolumn{3}{|c|}{ Présence des spermatozoïdes } & \multirow{2}{*}{ Côté } \\
\hline & & & Isthme & $\begin{array}{l}\text { I } / 2 \text { infér. } \\
\text { Ampoule }\end{array}$ & $\begin{array}{l}\text { I } 2 \text { supér. } \\
\text { Ampoule }\end{array}$ & \\
\hline & Vivants & $\begin{array}{l}5 \mathrm{I} 3 \\
839\end{array}$ & $\begin{array}{l}+ \\
+ \\
+ \\
+\end{array}$ & $\frac{+}{t}$ & $\bar{z}$ & $\begin{array}{l}\mathrm{G} \\
\mathrm{D} \\
\mathrm{G} \\
\mathrm{D}\end{array}$ \\
\hline In vitro & Morts & $\begin{array}{r}47 \\
44 \\
105 \\
195 \\
739\end{array}$ & $\begin{array}{l}- \\
+ \\
+ \\
+ \\
+ \\
+ \\
+ \\
+ \\
+ \\
+\end{array}$ & $\begin{array}{l}= \\
= \\
= \\
= \\
=\end{array}$ & $\begin{array}{l}\bar{z} \\
\bar{z} \\
\bar{z} \\
\bar{z} \\
=\end{array}$ & $\begin{array}{l}\mathrm{G} \\
\mathrm{D} \\
\mathrm{G} \\
\mathrm{D} \\
\mathrm{G} \\
\mathrm{D} \\
\mathrm{G} \\
\mathrm{D} \\
\mathrm{G} \\
\mathrm{D}\end{array}$ \\
\hline \multirow[b]{2}{*}{ In vivo } & Vivants & $\begin{array}{l}169 \\
15^{8} \\
141\end{array}$ & $\begin{array}{l}+ \\
+ \\
+ \\
+ \\
+ \\
+\end{array}$ & $\begin{array}{l}+ \\
+ \\
+ \\
+ \\
+ \\
+\end{array}$ & $\begin{array}{l}+\frac{1}{+} \\
\frac{+}{+}\end{array}$ & $\begin{array}{l}\mathrm{G} \\
\mathrm{D} \\
\mathrm{G} \\
\mathrm{D} \\
\mathrm{G} \\
\mathrm{D}\end{array}$ \\
\hline & Morts & $\begin{array}{r}53 \\
\text { I } 29 \\
5 \\
635 \\
5{ }^{12}\end{array}$ & $\begin{array}{l}\overline{-} \\
\overline{-} \\
\overline{+} \\
+ \\
+ \\
+ \\
\dot{+} \\
+ \\
+\end{array}$ & $\begin{array}{l}\bar{I} \\
\bar{I} \\
\bar{I} \\
\bar{I}\end{array}$ & $\begin{array}{l}\overline{ } \\
\bar{z} \\
\bar{z} \\
\bar{z} \\
\bar{z}\end{array}$ & $\begin{array}{l}G \\
\mathrm{D} \\
G \\
\mathrm{D} \\
\mathrm{G} \\
\mathrm{D} \\
\mathrm{G} \\
\mathrm{D} \\
\mathrm{G} \\
\mathrm{D}\end{array}$ \\
\hline
\end{tabular}


La fécondation d'œufs situés au-dessus de la zone inversée (tableau XXIV) prouve également que les spermatozoïdes de Bélier peuvent franchir les segments d'oviducte inversés, leur progression étant donc bien indépendante $\mathrm{du}$ sens des mouvements ciliaires. I,es essais négatifs observés par Kuo et Lim (I928) ne peuvent donc être attribués à une impossibilité pour les spermatozoïdes de franchir des fragments inversés.

Il y a donc un brassage général dû aux mouvements ciliaires et musculaires; toutefois, ce brassage dans la lumière des trompes ne peut suffire à expliquer la progression des spermatozoïdes. En effet, malgré le dépôt au bas de l'isthme d'un très grand nombre de spermatozoïdes morts, une infime proportion d'entre eux progresse dans la moitié supérieure de l'isthme, leur nombre étant le plus souvent inférieur à celui obtenu avec des spermatozoïdes vivants introduits seulement au sommet des cornes utérines. D'autre part, le niveau atteint par les spermatozoïdes morts est nettement plus éloigné de l'ovaire (tableau XXV), que celui oì sont dénombrés les spermatozoïdes vivants déposés pourtant plus bas.

Le facteur essentiel de la montée des spermatozoïdes dans cette partie du tractus paraît donc être la motilité propre des spermatozoïdes, les mouvements ciliaires ou musculaires n'intervenant que pour assurer un brassage général qui facilite leur dissémination.

\section{RÉSUMÉ. CONCLUSIONS}

L'observation méthodique de la montée des spermatozoïdes dans le tractus génital femelle nous a permis d'aboutir à la conclusion que la durée de transit des spermatozoïdes du vagin à la partie supérieure des trompes de Fallope de la Brebis et de la Vache est un phénomène relativement lent demandant environ 8 heures.

Les résultats obtenus par les différentes méthodes étant absolument concordants, on peut affirmer que la question de la durée de transit des spermatozoïdes dans le tractus génital femelle, qui a fait 1'objet de travaux nombreux et contradictoires, est définitivement tranchée; les migrations quasi instantanées, rapportées par certains chez les Ruminants, seraient très vraisemblablement dues à des erreurs expérimentales entrâ̂nant une pollution du matériel par des spermatozoïdes véhiculés accidentellement, comme nous en avons apporté la preuve directe.

L'analyse expérimentale des facteurs permettant ce transit a confirmé l'impossibilité physiologique d'une migration quasi instantanée. Ces facteurs nombreux, comme l'avaient laissé prévoir les travaux antérieurs, diffèrent, chez la Brebis, selon les niveaux du tractus.

La motilité propre des spermatozoïdes est indispensable pour le franchissement du cervix et de la jonction utéro-tubaire ; elle est néces- 
saire dans les trompes de Fallope où les mouvements ciliaires et les contractions musculaires sont également responsables de la diffusion des gamètes mâles.

Dans les cornes utérines, les contractions musculaires constituent le principal élément responsable de leur déplacement.

L'évaluation de la durée de transit des spermatozoïdes, chez la Brebis a permis la détermination exacte des durées du pouvoir fécondant des spermatozoïdes (3o heures) et de fécondité des ovocytes ( 15 heures).

Ce travail confirme ou précise, en outre, certains processus qui président à la rencontre des gamètes, et permettent une meilleure compréhension des phénomènes biologiques de la fécondation.

- Un très petit nombre des spermatozoïdes émis dans le vagin sont susceptibles d'atteindre l'œuf. Les zones du tractus oì la progression des spermatozoïdes est tributaire de leur motilité, limitent le passage des gamètes mâles et entraînent un gaspillage énorme.

- Ainsi, en dépit de la multitude de spermatozoïdes déposés dans le vagin, on conçoit, vu le petit nombre de gamètes mâles qui arrivent dans les trompes, que la montée des spermatozoïdes puisse être, aussi bien chez la Brebis que chez la Vache, un facteur limitant de succès d'une insémination. En effet, nous avons observé des cas de stérilité relative, la partie supérieure des trompes de Fallope n'étant pas atteinte par les spermatozoïdes. La connaissance des facteurs qui président à la progression des spermatozoïdes permet d'envisager une possibilité d'action sur chacun d'entre eux, et, par là même, une amélioration de la fertilité.

- La durée de survie des spermatozoïdes et des ovocytes laisse, par contre, une grande marge de sécurité dans la détermination du momnet de l'insémination.

\section{REFÉRENCES BIBLIOGRAPHIQUES}

Abarbanel, (A. R.). - Spermatozoa and cervical mucus. Dans Engle, The problem of fertility, I vol. Pinceton Univ. Press, II9-I33, I946.

Adams (C. E.). - A study of fertilization in the Rabbit : the effect of postcoital ligation of the fallopian tube or uterine horns. J. Endocrinol, 13, 296-308, 1956.

Adolphi (H.). - Die Spermatozoen der Säugetiere schwimmen gegen den Strom. Anat. Anz., 26, 549-559, I905.

AIDEN. - The utero-tubal junction in the albino rat. Anat. Rec., 85, 29029I, I943.

Ambache (N.), Hammond (J.jr). - Activity and drug responses of the Sheep uterus in relation to reproductive condition. $J$. Physiol., 108, 270-277, I949.

ANDERSEN (D. H.). - Comparative anatomy of the tubo-uterine junction. Histology and physiology in the Sow. Amer. J. Anat., 42, 255-305, I 928 . 
ANDERSON (J.). - Further investigations on artificial insemination of Sheep. J. Agric. Sci., 31, 354-369, I941.

Aspeli (S. A.), Salisbury (G. W.). - The viability of spermatozoa in the abdominal epididymis and the failure of motile sperms to fertilize ova. Amer. J. Physiol., 132, 79I-795, I94I.

Austin (C. R.). - Fertilization and the transport of gametes in the pseudopregnant Rabbit. J. Endocrinol., 6, 63-70, 1949.

BERRANG (K.). - Schleimdurchdring ungstermögen der Spermien in vitro beim Rind. Berl. Munch. Tierarztl. Wschr., 65, 35, I952.

Beschlebnov (A. V.). - The optimal time of inseminating Cows during cestrus. Problemy Zhivotnovod., 2, 73-88, I938.

Bischоғғ (T. L. W.). - Entwicklungsgeschichte des Kaninchen Eis-Braunschweig, I842.

BLANDAU (R. J.). - On the factors involved in sperm transport through the cervix uteri in the albino Rat. Amer. J. Anat., 7\%, 253-272, I945.

Blandau (R. J.), MoNey (W. L.). - Observations on the rate of transport of spermatozoa in the female genital tract of the Rat. Anat. Rec., 90, 255-260, I944.

BLANDAU (R. J.), OdOR (L.). - The total number of spermatozoa reaching segments of the reproductive tract of the Rat. Anat. Rec., 103, 93IIO, I949.

Boyarsky (L. H.), Bayliss (H.), Casida (L. E.), Meyer (R. K.). - Influence of progesterone upon the fertility of gonadotrophin treated female Rabbits. Endocrinology, 41, 312, I947.

BRADEN (A. W. H.). - Distribution of sperm in the genital tract of the female Rabbit after coitus. Aust. J. Biol. Sci., 6, 693-7I6, I953.

Brewster (J. E.), MAY (R.), Cole (C. L.). - The time of ovulation and rate of spermatozoa travel in Cattle. Am. Soc. Anim. Prod. Proc., 33, 304310, I940.

Brouha (L.), Simmonnet (H.). - Influence dulavage par une solution physiologique sur le rythme spontané de l'utérus. $C$. $R$. Soc. Biol., I54I 55, I 927 .

BURCKHARDT (J.). - Sperm survival in the genital tract of the Mare. $J$. Agric. Sci., 39, 20I-203, I949.

Chang (M. C.). - Fertilization in relation to the number of spermatozoa in the fallopian tube of Rabbits. Ann. Obstet. Gynecol., \%, 9I8-925, I 95 I.

Chang (M. C.). - Fertilizability of rabbit ova and the effects of temperature in vitro on their subsequent fertilization and activation in vitro. $J$. exp. Zool., 121, 35I-382, I952.

Chang (M. C.), Pricus (G.). - Physiology of fertilization in Mammals. Physiol. Rev., 31, I-26, 195I.

CLARK (R. T.). - Studies on the physiology of the reproduction in the Sheep. II. The cleavage stages of the Ovum. Anat. Rec., 60, I35-I60, I934.

Ciark (A. J.), Knaus (H. H.), Parkes (A. S.). - Estrus variations of uterine activity in the Rat. J. Pharmacol. exper. therap., 26, 359, I925-I926.

Courrier (R.). - Cycle animal de la glande interstitielle du testicule des Cheiroptères. C. R. Soc. Biol., 88, I923.

Courrier (R.). - Ettude sur le déterminisme des caractères sexuels secondaires chez quelques Mammifères à activité testiculaire périodique. Arch. Biol., 3\%, I73, I927.

Cupps (P. T.), AsdezL (S. A.). - Changes in the physiology and pharmacology of the uterine muscle of the Cow in relation to the œstrous cycle. J. Animal. Sci., 3, 25I-359, I944.

Curtis (V.), Lambert (W. V.). - A study of fertility in Poultry. Poultry Sci., 8, I42-I50, I 929 . 
DaUzier (L.). - - Recherches sur les facteurs de la remontée des spermatozoïdes dans les voies génitales femelles (trompes de Fallope). Étude chez la Brebis. C. R. Soc. Biol., 149, I94I-I943, I955.

DAUZier (L.). - Recherches sur les facteurs de la remontée des spermatozoides dans les voies génitales femelles (cornes utérines). Eitude chez la Brebis. C. R. Soc. Biol., 149, I872-I874, I955.

DAUZIFR (L.). - Recherches sur les facteurs de la remontée des sperma tozoïdes dans les voies génitales femelles. Etude chez la Brebis (col de l'utérus). C. R. Soc. Biol., 14\%, I556-I558, I953.

Dauzier (L..), Wintenberger (S.). - Recherches sur la fécondation chez les Mammifères. La remontée des spermatozoïdes dans le tractus génital de la Brebis. C. R. Soc. Biol., 146, 67-70, I952.

Dauzier (L.), Wintenberger (S.). - Recherches sut la fécondation chez les Mammifères. Durée du pouvoir fécondant des spermatozoïdes de Bélier dans le tractus génital de la Brebis et durée de la période de fécondité de l'œuf après l'ovulation. C. R. Soc. Biol., 146, 660-663, I952.

DAUZIER (L.), WINTENBERGER (S.). - Recherches sur la fécondation chez les Mammifères. La remontée des spermatozoïdes dans le tractus génital de la Brebis en dioestrus et ancestrus. C. R. Soc. Biol., 146, 663-665, I952.

DAuzier (L.), Wintenberger (S.). - La vitesse de remontée des spermatozoïdes dans le tractus génital de la Brebis. Ann. Zootechnie, 1, I3-22, I952.

Dauzier (L.), Wintendererer (S.). - Analyse des conditions de la fécondation chez la Brebis. 2nd Insem. Congr. Phys. Path. Anim. Reprod. Artif. Ins., Copenhague, II3-I24, I952.

Dauzier (L.), DU MESNil DU Buisson (F.). - La remontée des spermatozoïdes du Verrat dans le tractus génital de la Truie en œstrus. $C . R$. Soc. Biol., 149, 76-79, I955.

DAuziér (L.), DU MESNiL DU Buisson (F.). - Les spermatozoïdes dans l'appareil génital femelle (étude chez la Brebis, la Vache, la 'Truie). $2^{\mathrm{e}}$ Congrès Mondial de la Fertilité et de la Stérilité, Naples, I956 (sous presse), I956.

DAY (F. T.). - Survival of spermatozoa in the genital tract of the Mare. Agric. Sci., 32, I08-I II, I942.

Dewitz (J.). - Was veranlasst die Spermatozoen, in das Ei zu dringen. Arch. f. Anat. u. Physiol., Physiol. Abt., Ioo, Igo3.

Dickinson (R. L.). - The control of contraception. I vol. William and Wilkins, Baltimore, I93I.

EiLFORD (F. C.). - Report from the Poultry division. Rep. exp. Fms. Can., III6, I9I5.

Evans (E. J.). - The transport of spermatozoa in the Dog. Amer. J. Physiol., 105, 287-293, I933.

Evans (E. J.), Milier (F. W.). - Uterine motility in the Cow. Amer. J. Physiol., 116, 44-45, I936.

Faris (E. J.). - Studies of reproduction in the albino Rat. Anat. Rec., 84, 454 (abstract), I942.

Feldman (W. M.). - The principles of ante-natal and post-natal child physiology, pure and applied. I vol. (London), I920.

FERIN (J.). - Facteurs hormonaux et migration des spermatozoïdes dans l'utérus chez la Femme. Ann. Endocrinol., 9, 77-83, I948.

FLoREY (H.), WALTON (A.). - Uterine fistula used to determine the mechanism of ascent of the spermatozoa in the genital tract. Proc. Physiol. Soc. J. Physiol., 74, 5 pp., I932.

Fuente (F. de la). - La motilité de la trompe de Fallope in vitro. Rev. Fr. Gynecol. Obstetr., 78, 256-264, 195I. 
GENELL (S.). - Uterin-och vaginalmuskulaturens funktionella uppgifter iden icke-gravida orgamsmen-Ivol. gleerup-Lund (I937)

Gilbert (A. G.). - Report of the Poultry manager. Rep. exp. Fms. Can., 300, I 905 .

GREEN (W. W.), Winters (L. M.). - Studies on the reproduction in the Sheep. II. The time of ovulation and rate of sperm travel. Anat. Rec., 61, 457-470, I935.

GunN (J. A.). - The action of adrenaline and of choline-esters on the uterus of the Sheep. J. Physiol., 103, 290-296, I944.

HAMmoND (J.). - The fertilization of Rabbit ova in relation to time: a method of controlling the litter size, the duration of pregnancy and the weight of young at birth. J. exper. Biol., 11, I40-I6I, I934.

Hammond (J.). - Recent scientific research on Horse breeding problems. Trans. Yorks. Agric. Soc., 95, II, I938.

Hammond (J.), Asdest (S. A.). - The vitality of the spermatozoa in the female and the male reproductive tract. Br. J. exp. Biol., 4, I55-I85, I926.

Hammond (J.), Warton (A.). - Notes on ovulation and fertilization in the Ferret. J. exp. Biol., 11, 307-327, I934.

HaRTMAN (C. G.), BaLL (J.). - On the almost instantaneous transport of spermatozoa through the cervix and the uterus in the Rat. Proc. Soc. exp. Biol. Med., 28, 3I2-3I4, I930.

HAys (R. L.), VAN Demark (N. L.). - Stimulatory action of breeding on the release of oxytocin as measured by intrammary pressure. J. Dairy Sci., 34, 496, I95I,

Hays (R. L.), VAN Demark (N. L.). - Uterine motility responses to mating. Amer. J. Physiol., 170, 5I8-52I, I952.

Hays (R. I.), VAN Demark (N. L.). - Spontaneous motility of the Bovine uterus. Amer. J. Physiol., 172, 553-556, I953.

Hays (R. L.), VAN DEMark (N. L.). - Effects of oxytocin and epinephrine on uterine motility in the Bovine. Amer. J. Physiol., 1ra, 557-56o, I953.

Hays (R. L.), VAN DEMARK (N. I.). - Effect of stimulation of the reproductive organs of the Cow on the release of an oxytocin like substance. Endocrinology, 52, 634-637, I953.

HEAPE (W.). - The artificial insemination of Mares. Veter., \%1, 202, I898.

Heape (W.). - Ovulation and degeneration of ova in the Rabbit. Proc. Roy. Soc., Ser. B, 76, 260-268, I905.

HENSEN (V.). - Beobachtungen über die Befruchtung und Entwicklung des Kaninchens und Meerschweinchens. Zeitschr. Anat. Entwick, 1, 213273, 1876 .

Hozhne (O.). - Ueber die Flimmerung im Genitalapparat mit bes, Berïcksichtigung des Eitransports. Verhandl. deutsch. Ges. Geburtsh. Gynäk., $8 \mathrm{I} 7$, IgII.

HUEHNER (H.). - Sterility in the male and female, and its treatment. $x$ vol. Retman Co, New York, I9I3.

KeILEY (R. B.). - Studies in fertility of Sheep. Bull. Coun. Sci. industr. Res. Austr., $\mathrm{n}^{\circ} \mathrm{II} 2, \mathrm{I} 937$.

KeLLy (G. L.). - The utero tubal junction in the Guinea-pig. Amer. J. Anat., 40, 373-385, I 928 .

McKenzie (F. F.), Terrill (C. E.). - CEstrus, ovulation and related phenomena in the Ewe. Res. Bull. Agric. St. Missouri, no 264, 88 pp., I937.

KING (J. L.). - Observations on the activity and working power of the uterine muscle of the non pregnant Sow. Amer. J. Physiol., 81, 725-737, I927.

KiRILIOV (V.). - The organization of two Lamb crops in a year. Sovhoz. Proizyvod., 12, 34, 1944 . 
Kozlova (V. M.). - Doses optimales du sperme eritier au cours de l'insémination des Vaches. Problèmes de l'élevage, $\mathrm{n}^{\circ} 45$, I935.

Krehbiei (R. H.), Carstens (H. P.). - Roentgen studies of the mechanism involved in sperm transportation in the female Rabbit. Amer. J. Physiol., 125, 57I-577, I939.

KUO (Y. U.), LIM (R. K. S.). - On the mechanism of the transportation of ova. Chin. J. Physiol., 2, 389-398, I928.

LAING (J. A.). - Observations on the survival time of the spermatozoa in the genital tract of the Cow and its relation to fertility. J. Agric. Sci., 35, 72-83, I 944 .

LAING (J.A.). - Observations on the characteristics of the semen in relation to fertility in the Bull. J. Agric. Sci., 35, I, I945.

Lamar (J. K.), ShetTles (L. B.), Delfas (E.). - Cyclic penetrability of human cervical mucus to spermatozoa in vitro. Amer. J. Physiol., 129, 234, I940.

LEE (F. C.). - The tubo-uterine junction in various animals. Bull. Johns Hopkins Hosp, 42, 335-357, I928.

LEONARD (S. L.). - The reduction of uterine sperm and uterine fluid in fertilization of Rat ova. Anat. Rec., 106, 607-6I5, I950.

Leonard (S. L.), Periman (P. L.). - Conditions affecting the passage of spermatozoa through the utero tubal junction of the Rat. Anat. Rec., 104, 89-I02, I949.

LEUCKARDT (R.). - Zeugung. In Wagner's Handwörterbuch der Physiologie, 4, 707, I853.

IEWIS (W. H.). - The vitality of reproductive cells. Bull. Okla. Agric. exp. Sta., 96, 3, I9II.

LEWIS (W. H.), WRIGHT (E. S.). - On the early development of the Mouse egg. Contr. Embryol. Carnegie Inst., 25, II3-I4I, I935.

LIM (R. K. S.), ChaO (C.). - On the mechanism of the transportation of ova. I. Rabbit uterus. Chin. J. Physiol., 1, I75-I98, I927.

LIM (R. K. S.), Chao (C.). - Observations on the " reversed " uterine horn of the Rabbit. Proc. Soc. exp. Biol. Med., 23, 668-669, r926.

LLOYD-JONES (O.), HAYS (F. A.). - The influence of excessive sexual activity of male Rabbits. I. On the properties of the seminal discharge. $\bar{J}$. exp. Zool., 25, 463, I9I8.

LoNg (J.A.), Evans (H. M.). - The cestrous cycle in the Rat and its associated phenomena. Mem. Univ. Calif., 6, I-I48, I922.

LOPYRIN (A. I.), I,OGINOVA (N. V.). - Vitesse de remontée et temps de survie des spermatozoïdes dans le tractus génital de la Brebis. Sovetsk, Zootech., $\mathrm{n}^{\circ} \mathbf{2 , 3}$, I44-I 49, I939.

LotT $(\mathrm{G}$.$) . - Zur Anatomie und Physiologie des Cervix uteri. F. Enke, Er-$ langer, 9, I50 pp., I872.

Low (O.). - Die Chemotaxis des Spermatozoen im weiblichen genital tract. Sitz. Berich. Hathen. Naturwiss. Class., 111, II8-I82, I902.

Ludwig (C.). - Lehrbuch der Physiologie des Menschen. (Leipzig), I86I.

MARShali (F. H. A.). - The physiology of reproduction. I vol. Longmans, Green and Co, London, 867 pp., I922.

MARTIN (A.). - Handbuch der Krankheiten der weiblichen Adnexorgane. (Leipzig), I895.

Merton (H.). - Studies on reproduction in the albino Mouse. II. Contributions on the maturation of the sperm cells. Proc. Roy.Soc., Sér. B, 59, I45-I52, I939.

Mimura (H.). - Studies on the mechanism of travel of spermatozoa through the oviduct in the domestic female Fowl. J. Dept. Agric. Univ. Imp. Kiou Sion, 5, 265-269, r94I. 
Murphee (R. I.), Biack (W. G.), Ot'To (G.), Casida (L. E.). - Effect of site of insemination upon the fertility of gonadotrophin treated Rabbits of different reproductive stages. Endocrinology, 49, 474-480, I95I.

Murphee (R. I.), Warwick (E. J.), Casida (L. E.), McShan (W. H.). Influence of reproductive stage upon the fertility of gonadotrophin treated female Rabbits. Endocrinology, 41, 308-3II, I947.

Nicolaides (C.). - Fertility studies in Poultry. Poultry Sci., 13, I78-I83, I934.

Or'Tavant (R.), Thibault (C.), Wintenberger (S.). - Contribution à l'étude de la superovulation expérimentale chez la Brebis. Ann. Endocrinol., 10, I70-I73, I949.

PARkER (G. H.). - The passage of spermatozoa and ova through the oviducts of the Rabbit. Proc. Soc. exp. Biol. Med., 27, 826, I930.

PARker (G. H.). - The passage of sperms and of eggs through the oviducts in terrestrial vertebrates. Philos. Trans. Roy. Soc. London, Sér. B, 219, 38I-4I9, I93I.

Philips (A. G.). - Brief study of the mating of Fowls with a test of the value of a single mating. J. Amer. Assoc. Inst. and Invest. of Poultry Husb., 4, 30, I9I8.

Phillips (R. W.), ANDREWs (F. N.). - The speed of travel of the Ram spermatozoa. Anat. Rec., 68, I27-I32, I937.

Polovceva (V. V.). I938. - Dokl. Akad. seljskohoz Nank, 43 I5-I6, dans IAING (J. A.), I944,

Pommerenke (W. T.), Viergiver (E.). - Cervical mucus and the menstrual cycle. In The problems of fertility, Engle. London, IO2-II8, I946.

PoMpen (A. W. H.). - De Invloed van Menformon op de Baarmoeder. Thesis, Amsterdam, I933.

Quinlan (J.), Mare (G. S.), Roux (L. L.). - The vitality of the spermatozoa in the genital tract of the Merino ewe, with special reference to its practical application in breeding. XVIIIth Rep. Direction Vet. Ser. Anim. Indust. Union of South Africa, 2, 83I-870, I932.

REDENZ (E.). - Das Verhalten der Säugetierspermatozoen zwischen Begattung und Befruchtung. Z. Zellforschr. Mikros. Anat., 9, 734, I929.

REYNolds (S. R.). - Studies on the uterus. I. A method for recording uterine activity in chronic experiments on unanesthetized animals. Amer. J. Physiol., 92, 420-429, 1930.

Reynolds (S. R.). - Physiology of the uterus. 2nd ed. Paul B. Hoeber, Inc. New York, 567 pp., I949.

REYNolds (S. R.), FRIEDMAN (M. H.). - Studies on the uterus. II. Ithe activity of the uterine fistula in unanesthetized rabbit following coitus and during pseudopregnancy. Amer. J. Physiol., 94, 696, I930.

Robinson (I. J.). - Reproduction in the Ewe. Biol. Rev., 26, I2I-I57, I95 I.

Rossman ("T. J.). - - Uterine contractions and the transport of sperm in the rat. Anat. Rec., 79, I33-I49, I937.

Rowson (L. E.). - The movement of radioopaque material in the bovine uterine tract. Brit. vet. J., 111, 334-342, I955.

SAGER (V.), LEONARD (S. L.). - Relation of oestrin and pregnancy urine hormone in influencing uterine motility. Proc. Soc. exp. Biol. Med., 35, 242, 1936 .

Salisbury (G. W.), Van Demark (N. L.). - - Physiological basis for site of semen deposition and procedure in artificial insemination of Cattle. The IIth Intern. Congress. Physiol. Path. Animal Reprod. Artif. Insem. Copenhagen, 99-106, 1952.

SAN MaRTin (F. M.). - Some experiments on reproduction activity. Thesis, Fac. Med. Univ. Nac. Mayor San Marcos, Lima Gopp. (A. B. A., 20, 257, I952), I95I. 
Schot (R. G.), Philims (R. W.). - Rate of sperm travel and the time of ovulation in Sheep. Anat. Rec., 79, 531-540, I94I.

SECKINGER (D. L.). - Spontaneous contractions of the fallopian tubes of the domestic Pig with reference to the oestrous cycle. Bull. Johns Hopkins Hosp., 34, 236, I923.

Seguy (J.), Vimeux (J.). - Contribution à l'étude des stérilités inexpliquées : étude de l'ascension des spermatozoïdes dans les voies génitales basses femelles. Gynecol. et Obstetr., 2\%, 346-358, I934.

Srmms (J. M.). - Clinical notes on uterine surgery. William Wood and Co, New York, I 886.

Skatkine (P. N.), Rumjantzewa (E. U.). - Vitesse de déplacement des spermatozoïdes dans les organes femelles sexuels. Rap. Acad. Sov. Lénine Sc. Agron., 10, I948.

SODERWALL (A. L.), BI.ANDAU (R. J.). - The duration of the fertilizing capacity of spermatozoa in the female genital tract of the Rat. J. Exp. Zool., 88, 55-63, I94I.

STARKE (N. C.). - The sperm picture of Rams of different breeds. II. The rate of sperm travel in the genital tract of the Ewe. Ondersteport $J$. Vet. Sci. and Anim. Ind., 22, 4I5-425, 1949.

StErNach (E.). - Untersuchungen zur vergleichenden Physiologie der accessorischen Geschlechtsdriisen. Pffugers Arch., 56, 304-334, I894.

TAlmey (B. S.). - Birth control and the physician. New York Med., 5, 105, II85, I9I7.

Thibaurit (C.). - L'œuf des mammifères. Son développement parthénogénétique. Ann. Sc. Nat. Zool., Ser. XI, I36-219, I949.

Thibault (C.), Ortavant (R.), Iaplaud (M.). - Recherches sur la superovulation expérimentale chez la Brebis. Ann. Endocrinol., 9, 83-89, I 948.

Thibault (C.), Ortavant (R.), Laplaud (M.). - Utilisation du nesdonal comme anesthésique chez le Mouton. Comparaison de son action avec celle de l'évipan et du chloral. C. R. Soc. Biol., 142, 20-22, I 948.

Trimberger (G. W.), Davis (H. P.). - Conception rate in dairy Cattle by artificial insemination at various stages of oestrus. Res. Bull. Neb. Agric. exp. Sta., no I29, I943.

VAN Demark (N. L.). - Motility patterns in the female reproduction tract. J. Sci. Iowa State, 28, I07-II8, I953.

VAN Demark (N. I..). - Spermatozoa in the female genital tract. $2^{\text {e }}$ Congrès Mondial de la Fertilité et de la Stérilité, Naples, I956, sous presse, I956.

VAN Demark (N. L.), Hays (R. L.). - Uterine motility responses to mating. Amer. J. Physiol., 170, 5I8-52I, I952.

VAN Demark (N. L.), Mofil.ER (A. N.). - Spermatozoan transport in the reproductive tract of the Cow. J. Dairy Sc., 33, 390-39I, I950.

VAN Demark (N. L.), MOELLER (A. N.). - - Speed of spermatozoa transport in reproductive tract of oestrous Cow. Amer. J. Physiol., 165, 674$679, \mathbf{I} 95 \mathrm{I}$.

VAndesplache (M.), Paredis (E.). - Preservation of the fertilizing capacity of Bull semen in the genital tract of the cow. Nature, 162, 813, I948.

Van Drimmelen (G. C.). - Artificial breeding of Poultry. The J. of South Africa. Vet. Med. Assoc., 18, 39-44, 1947.

WALKER (G.). - The effect on breeding of the removal of the prostate gland or of the seminal vesieles, or of both together with observations on the conditions of the testes after such operations on white Rats. Johns Hopkins Hosp. Rep., 16, 223-255, IgII.

WaITON (A.). - The effect of temperature on the survival in vitro of Rabbit spermatozoa obtained from the was deferens. Br. J. exp. Biol., 7, $\mathrm{n}^{\circ} 2$, I930. 
WaLTON (A.). - Flow orientation as a possible explanation of "wave motion " and "rheotaxis" of spermatozoa. J. exp. Biol., 29, 520-53I, I952.

WARREN (M. R.). - Observations on the uterin fluid of the Rat. Amer. $J$. Physiol., 122, 602-608, I938.

Westman (A. A.). - A contribution to the question of the transit of the ovum from the ovary to the uterus in Rabbits. Acta Obst. Gynec. Scand., 5, 7, I926.

Westman (A.). - Untersuchungen über die Physiologie der Tuba uterina bei Macacus. thesus. Acta. Obst. Gynecol. Scand., 8, 307, I929.

Whitney (L. F.). - The mating cycle of the Dog. Chase Mag., rg27.

WiJSENBECK (I. A.), GREVENSTUK (A.). - Over de bewegingen van de barrmoeder. (waarnemingen aan het zwangere, barende en puerperale orgaan). Nederl. Tijdschr. Geneesk., 66, 2155-2164, I922.

WINTENBERGER (S.). - Recherches sur les relations entre l'œuf et le tractus maternel pendant les premiers stades du développement chez les mammifères. Étude de la traversée de l'oviducte par l'œuf fécondé de Brebis. Ann. Zootechnie, 3, 269-273, I953.

Wislocki (G. M.), GUtTMacher (A. F.). - Spontaneous peristaltis of the excised uterus and fallopian tubes of the Sow with reference to the ovulation cycle. Bull. Johns Hopkins Hosp., 35, 246-252, I924.

YAMANE (J.), ITo (T.). - Ueber die Geschwindigkeit der Pferdespermatozoen in stromenden und nichtstromenden Flussigkeiten, $\mathbf{I} 932$.

Yochem (D. E.). - Spermatozoon life in the female reproductive tract of the Guinea pig and the Rat. Biol. Bull., 56, 274-297, I939. 